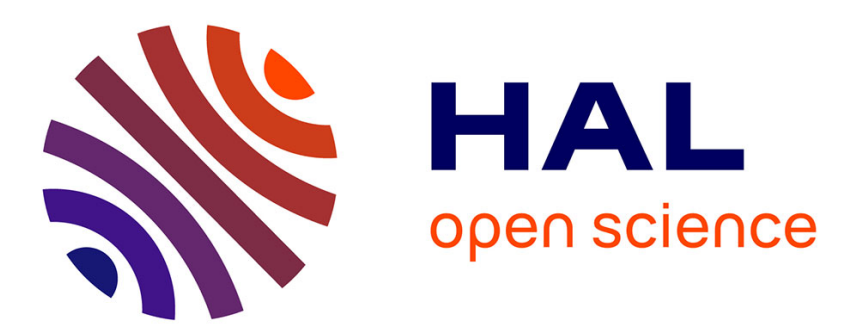

\title{
Numerical modelling of crack propagation in ductile materials combining the GTN model and X-FEM
}

\author{
Jean-Philippe Crété, Patrice Longère, Jean-Marc Cadou
}

\section{To cite this version:}

Jean-Philippe Crété, Patrice Longère, Jean-Marc Cadou. Numerical modelling of crack propagation in ductile materials combining the GTN model and X-FEM. Computer Methods in Applied Mechanics and Engineering, 2014, pp.204-233. 10.1016/j.cma.2014.03.007 . hal-01113127

\section{HAL Id: hal-01113127 \\ https://hal.science/hal-01113127}

Submitted on 19 Jun 2018

HAL is a multi-disciplinary open access archive for the deposit and dissemination of scientific research documents, whether they are published or not. The documents may come from teaching and research institutions in France or abroad, or from public or private research centers.
L'archive ouverte pluridisciplinaire HAL, est destinée au dépôt et à la diffusion de documents scientifiques de niveau recherche, publiés ou non, émanant des établissements d'enseignement et de recherche français ou étrangers, des laboratoires publics ou privés. 


\title{
Numerical modelling of crack propagation in ductile materials combining the GTN model and X-FEM
}

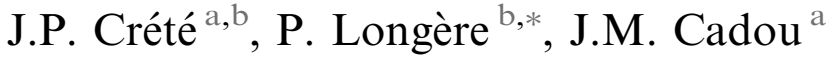 \\ ${ }^{a}$ Université de Bretagne Sud, LIMATB (EA 4250), Lorient, France \\ ${ }^{\mathrm{b}}$ Université de Toulouse, ISAE/ICA (EA 814), Toulouse, France
}

The present work is devoted to the numerical simulation of crack propagation in engineering materials whose failure results from void initiation, growth and coalescence. The behaviour of the plate material is described via a Gurson type model accounting for the combined effects of strain hardening, thermal softening, viscoplasticity and void growth induced damage. The eXtended Finite Element Method has been retained to describe the kinematic consequences of the crack propagation across the mesh. The crack is assumed to propagate as soon as the stored energy around the crack tip reaches a critical value. The related crack length is estimated using an exhaustion method. The constitutive model and the extended finite elements were both implemented in the engineering FE computation code Abaqus as user subroutines. The numerical simulation of a notched plate and an asymmetrically notched plate under tension loading has been conducted. While making some simplifications, the present work reproduces numerically the $2 \mathrm{D}$ propagation of a crack resulting from void growth induced damage.

Keywords: EXtended Finite Element Method; Ductile failure; Porous materials

\section{Introduction}

The optimisation of metal cutting conditions and the prediction of the residual strength of overloaded metallic structures require reliable numerical simulations of crack propagation in and further ultimate ruin of engineering materials whose failure mostly results from void growth induced damage. Achieving this goal implies taking up several challenges. Among others are particularly asked the questions of (i) the pertinence of the constitutive model supposed to be representative of the behaviour of the material in presence of the damage mechanism leading to fracture, (ii) the accuracy of the indicator allowing for passing from diffuse damage

\footnotetext{
* Corresponding author. Tel.: +33 56133 81 15; fax: +33 561339095 .

E-mail address: patrice.longere@isae.fr (P. Longère).
} 
to crack formation, (iii) the reliability of the technique employed for solving the boundary value problem involving crack propagation, and (iv) the tractability of the methodology developed into engineering computation codes for industrial applications.

The fracture of ductile materials is known to result from void initiation, growth and coalescence. During the process of ductile damage, the bulk material is subject to a progressive loss of its overall properties and to the appearance of an inelastic dilatancy due to void growth, in addition to the isochoric plastic deformation due to dislocation glide in the matrix material. Since the pioneering works by Berg in the late 1960's, many attempts have been done for describing the aforementioned ductile damage effects, see e.g., Gurson [1], Perzyna [2], Lemaitre [3], Rousselier [4], Brünig [5]. Due to its ability for the prediction of fracture of metals under tension loading, the micromechanics based Gurson's model has progressively become the probably most referenced model in ductile fracture, see e.g., Tvergaard and Needleman [6], Becker et al. [7], Leblond et al. [8], Nahshon and Hutchinson [9], Longère et al. [10]. The wide use of the so-called GTN model (for Gurson, Tvergaard and Needleman, see Tvergaard and Needleman [6]) for predicting the strain localisation conditions in damage induced softening metallic materials must also be noted, see e.g., Yamamoto (1978), Mear and Hutchinson [11], Besson et al. [12].

The numerical treatment of crack propagation in engineering structures is far from being trivial. When the standard finite element formulation (FEM) is employed, singularities of the stress-strain fields at the crack tip require a very fine meshing in the vicinity of the crack tip whereas the other parts of the structure can be more coarsely meshed. This implies meshing finely the area covered by the crack during its propagation (which supposes knowing a priori the crack path) or using an adaptative meshing technique, see e.g., Bouchard et al. [13]. Both aforementioned methods are however expensive in terms of computation cost. In the late of 1990's, the idea of embedding the crack induced strong discontinuity into the finite element has emerged and given birth to the so-called eXtended Finite Element Method (X-FEM), see e.g., Moes et al. [14]. Based on the partition of unity concept, see Melenk and Babuska [15], the latter consists in accounting for the kinematics induced by the crack propagation by adding supplementary degrees of freedom in the finite element formulation. X-FEM has been since widely used to reproduce the failure of structures consisted mostly of quasi brittle materials, see e.g., Moës et al. [14], Grégoire et al. [16], whereas its applications to ductile fracture remain few in literature, see the very recent works by Haboussa et al. [17,18], Pourmodheji and Mashayekhi [19], Seabra et al. [20], Broumand and Khoei [21].

Combining a ductile damage material constitutive model and the eXtended Finite Element Method requires criteria allowing for passing from continuous damage mechanics to (non linear) fracture mechanics. When brittle materials are involved, according to linear fracture mechanics, it is possible to use as crack propagation indicators the critical value of the (local) stress intensity factor K or, better, the one of the (global) Griffith energy release rate $\mathrm{G}$. This is no longer possible when materials exhibit strongly non linear behaviour (as it is the case in the present work). Damage induced softening and/or non radial loading paths moreover prevent considering the Rice-Cherepanov J-Integral as possible crack propagation indicator. When local crack propagation indicators involving internal variables (such as e.g., a critical void volume fraction) at the crack tip are used, the issue concerning the mesh dependence has also to be considered. The lack of time resolved experimental data during this transition between (more or less) diffuse damage and crack formation makes the problem even more complicated to describe. Hypotheses regarding the microscopic underlying mechanisms leading to crack incipience, as well as simplifications for the numerical treatment of the crack propagation, have consequently to be made.

We propose in the present work a methodology combining a modified Gurson based, finite strain, (ductile) damage - (visco) plasticity coupled constitutive model and the eXtended Finite Element Method (X-FEM), both implemented into the engineering computation code Abaqus (see Giner et al. [22] for example). The GTN model version considered in the present work has been proposed by Longère et al. [10] to reproduce the void growth induced damage in shear. Motivated by physics, the modification consists in shifting the GTN yield locus and plastic potential, allowing inelastic dilatancy (according to normality rule) and further void growth under low negative stress triaxiality loadings, see also Longère and Dragon [23]. The model implemented as user material subroutine (umat) is outlined in Section 2. Concerning the X-FEM formulation, the technique consisting in increasing significantly the number of integration points, see Elguedj et al. [24], of the original finite element has been retained here to capture the crack propagation. The X-FEM based 
approximation used for reproducing the crack propagation via a user finite element subroutine (uel) is described in Section 3. To attenuate mesh dependence, the indicator of the transition from continuous damage to crack formation considers quantities averaging over an area (a patch) located at the crack tip, as applied by Haboussa et al. [17]. The crack is assumed here to initiate as soon as an averaged, stored energy related quantity around the crack tip reaches a critical value. In the line of the works by Huespe et al. [25], the crack formation is assumed to result from a deformation localisation process and its orientation is accordingly deduced from the bifurcation analysis (in the sense of [34]). The corresponding procedure, which clearly constitutes the core of the present work, is detailed in Section 4. Some applications considering a notched plate and an asymmetrically notched plate submitted to tension loading are finally shown in Section 5.

\section{Material constitutive model}

In the present work, materials considered are metals and alloys whose failure is known to result from void growth induced damage, see e.g., Longère et al. [10]. The damage process (before crack formation) is consequently isotropic in nature, and it is accordingly pertinent to account for the current material damage state via a scalar variable.

The constitutive model, accounting for strain hardening, thermal softening and void growth induced damage, in view of describing the material deformation process under low and high strain rate loadings is outlined in the sequel.

\subsection{Thermodynamic framework}

We are here considering a metallic material subject to thermal softening, isotropic hardening, and ductile damage. The state variables to account for are accordingly: the elastic strain tensor $\epsilon_{\sim}^{e}$, the absolute temperature $T$, the isotropic hardening variable $\kappa$ and the damage related variable $D$.

The instantaneous material state is supposed to be well described via the Helmholtz free energy $\omega\left(\epsilon_{\sim}^{e}, T, \kappa, D\right)$ which is decomposed, as classically done, into reversible, stored, thermal and damage parts, namely $\omega_{r}\left(\epsilon_{\sim}^{e}, T, D\right), \omega_{s}(\kappa, T, D), \omega_{T}(T, D)$ and $\omega_{D}(D)$, respectively:

$$
\omega\left({\underset{\sim}{\epsilon}}_{\epsilon}^{e}, T, \kappa, D\right)=\omega_{r}\left(\epsilon_{\sim}^{e}, T, D\right)+\omega_{s}(\kappa, T, D)+\omega_{T}(T, D)+\omega_{D}(D)
$$

The state variables conjugate to $\left(\epsilon_{\sim}^{e}, T, \kappa, D\right)$ are the thermo-elastic stress tensor $\underset{\sim}{\sigma}$, entropy $\eta$, isotropic hardening force $r$ and damage related force $Y$, such that

$$
\begin{aligned}
& \underset{\sim}{\sigma}=\frac{\partial \omega}{\partial \epsilon_{\sim}^{e}}=\frac{\partial \omega_{r}}{\partial \epsilon_{\sim}^{e}} \\
& -\eta=\frac{\partial \omega}{\partial T}=\frac{\partial \omega_{r}}{\partial T}+\frac{\partial \omega_{s}}{\partial T}+\frac{\partial \omega_{T}}{\partial T} \\
& r=\frac{\partial \omega}{\partial \kappa}=\frac{\partial \omega_{s}}{\partial \kappa} \\
& -Y=\frac{\partial \omega}{\partial D}=\frac{\partial \omega_{r}}{\partial D}+\frac{\partial \omega_{s}}{\partial D}+\frac{\partial \omega_{T}}{\partial D}+\frac{\mathrm{d} \omega_{D}}{\mathrm{~d} D}
\end{aligned}
$$

For the present purpose, the dissipation potential $\Phi(\underset{\sim}{\sigma}, r, Y ; T, D)$ may be written from a partition into damage-plastic and purely damage contributions, namely $\Phi_{P}(\underset{\sim}{\sigma}, r ; T, D)$ and $\Phi_{D}(Y ; T, D)$, respectively:

$$
\Phi(\underset{\sim}{\sigma}, r, Y ; T, D)=\Phi_{P}(\underset{\sim}{\sigma}, r ; T, D)+\Phi_{D}(Y ; T, D)
$$

In the irreversible thermodynamics based Lemaitre model, used in [19], thermal softening is not accounted for, and strong damage state coupling - weak damage-plasticity kinetic coupling are assumed in the context of generalised standard material, leading to 


$$
\begin{aligned}
& \omega\left({\underset{\sim}{\epsilon^{e}}}^{e}, \kappa, D\right)=\omega_{r}\left({\underset{\sim}{\epsilon}}_{\sim}^{e}, D\right)+\omega_{s}(\kappa) \\
& \Phi(\underset{\sim}{\sigma}, r, Y ; D)=\Phi_{P}(\underset{\sim}{\sigma}, r)+\Phi_{D}(Y ; D)
\end{aligned}
$$

Conversely, the micromechanics based Gurson model $(D=f)$ implicitly assumes weak damage state coupling and strong damage-plasticity kinetic coupling (standard material), yielding

$$
\begin{aligned}
& \omega\left(\underset{\sim}{\epsilon_{\sim}^{e}}, \kappa\right)=\omega_{r}\left({\underset{\sim}{\epsilon}}^{e}\right)+\omega_{s}(\kappa) \\
& \Phi(\underset{\sim}{\sigma}, r ; D)=\Phi_{P}(\underset{\sim}{\sigma}, r ; f)
\end{aligned}
$$

In the following, we are assuming that ductile damage does not affect much the elastic and plastic material properties but modifies significantly the yielding condition and direction. In other words, we are considering materials whose behaviour obeys Gurson type yield locus and plastic potential.

\subsection{Constitutive equations}

According to the assumption of a weak damage state coupling, and accounting for the effect of plastic dissipation induced thermal softening on strain hardening, the various contributions of the state potential $\omega\left(\epsilon_{\sim}^{e}, \kappa, T\right)$ are expressed by

$$
\begin{aligned}
& \omega_{r}\left({\underset{\sim}{e}}^{e}\right)=\frac{1}{2} \underset{\sim}{\epsilon^{e}}: \underset{\approx}{C}: \epsilon_{\sim}^{e} \\
& \omega_{s}(\kappa, T)=h(\kappa) g(T)
\end{aligned}
$$

where $\underset{\approx}{C}$ represents the isotropic linear elastic stiffness fourth order tensor, $h(\kappa)$ the stored energy of cold work and $g(T)$ the thermal softening function. The elastic stress and isotropic hardening force are consequently written as

$$
\begin{aligned}
& \underset{\sim}{\sigma}=\frac{\partial \omega}{\partial \epsilon_{\sim}^{e}}=\frac{\mathrm{d} \omega_{r}}{\mathrm{~d} \epsilon_{\sim}^{e}}=\underset{\approx}{C}: \underset{\sim}{\epsilon_{\sim}^{e}} \\
& r=\frac{\partial \omega}{\partial \kappa}=\frac{\partial \omega_{s}}{\partial \kappa}=h^{\prime}(\kappa) g(T)
\end{aligned}
$$

The (isotropic) strain hardening and thermal softening functions are assumed in the forms of a Voce type law and a power law, respectively:

$$
\begin{aligned}
& h^{\prime}(\kappa)=R_{\infty}[1-\exp (-k \kappa)]^{\beta} \\
& g(T)=1-\left(\frac{T}{T_{\text {ref }}}\right)^{m}
\end{aligned}
$$

where $\left(R_{\infty}, \mathrm{k}, \beta\right)$ are isotropic hardening related constants and $\left(T_{\text {ref }}, m\right)$ thermal softening related constants.

To reproduce the strong damage-plasticity kinetic couplings, the micromechanics based Gurson's model, see Gurson [1], modified by Tvergaard and Needleman [6], to better reproduce experimental results in tension, has been retained. Motivated by physical considerations, Longère et al. [23], recently introduced in the socalled GTN model a back mean stress provoking a shift of the yield locus towards negative stress triaxialities allowing to describe void growth under shear dominated loading. The resulting modified damage-plastic potential is accordingly proposed in the form

$$
\begin{aligned}
& \Phi=\left(\frac{\sigma_{e q}}{\overline{\sigma_{y}}}\right)^{2}+2 q_{1} f \cosh \left(-\frac{3}{2} q_{2} \frac{p_{m}+p_{r}}{\overline{\sigma_{y}}}\right)-\left(1+q_{3} f^{2}\right)=0 \\
& p_{r}=b \ln \left(q_{1} f\right)
\end{aligned}
$$

where $\sigma_{e q}$ represents the equivalent stress, $p_{m}$ the pressure, $p_{r}$ the back pressure (equals to the opposite of the aforementioned back mean stress), $\overline{\sigma_{y}}$ the rate dependent yield stress, $f$ the volume fraction of voids, and where 
$\left(q_{1}, q_{2}, q_{3}, b\right)$ are positive material constants. The rate dependent yield stress $\overline{\sigma_{y}}$ accounts for the combined effects of strain hardening, thermal softening and viscoplasticity:

$$
\overline{\sigma_{y}}(\kappa, \dot{\kappa}, T, \ldots)=\sigma_{y}(\kappa, T)+\sigma_{v p}(\dot{\kappa}, T, \ldots)
$$

where $\dot{\kappa}$ represents the matrix plastic strain rate. The rate independent yield stress $\sigma_{y}(\kappa, T)$ is written as

$$
\sigma_{y}=R_{0} g(T)+r(\kappa, T)
$$

where $R_{0}$ represents the initial radius of the Huber-Mises yield function at $0 \mathrm{~K}$. Injecting (14)-(16) into (20) yields

$$
\sigma_{y}=\left(R_{0}+R_{\infty}[1-\exp (-k \kappa)]^{\beta}\right)\left(1-\left(\frac{T}{T_{r e f}}\right)^{m}\right)
$$

The strain rate induced overstress $\sigma_{v p}(\dot{\kappa}, T, \ldots)$ reproduces as well the potential tension/compression asymmetry, see [26]:

$$
\sigma_{v p}=Y\left[\dot{\kappa} \exp \left(\frac{V_{a} p_{m}}{k_{b} T}\right)\right]^{\frac{1}{n}}
$$

where $(Y, n)$ are viscosity related constants and $\left(V_{a}, k_{B}\right)$ behaviour asymmetry related constants, with $V_{a}=V_{h} \beta^{3}$ where $V_{h}$ is a constant and $\beta$ Burgers vector magnitude $(\beta=2.5 \AA)$, and with $k_{B}$ Boltzmann constant $\left(k_{B}=1.3804 .10^{-23} \mathrm{~J} / \mathrm{K}\right)$.

The expression of the plastic strain rate $\dot{\epsilon}^{p}$, in the rotated frame (to be defined later), is deduced from the normality rule assumption:

$$
\begin{aligned}
& \dot{\epsilon}_{\sim}^{p}=\Lambda \underset{\sim}{\frac{\partial \Phi}{\sigma \sigma}}=\Lambda\left(\frac{\partial \Phi}{\partial \sigma_{e q}} \underset{\sim}{n}-\frac{1}{3} \frac{\partial \Phi}{\partial p_{m}} \underset{\sim}{\delta}\right)=\dot{\epsilon}^{p D} \underset{\sim}{n}+\frac{1}{3} \dot{\epsilon}^{p M} \underset{\sim}{\delta} \\
& \stackrel{n}{\sim}=\frac{3}{2} \frac{s \sim}{\sigma_{e q}}
\end{aligned}
$$

where $\underset{\sim}{s}$ represents the deviatoric part of the stress tensor $\sigma$ and $\delta$ the identity tensor. The distortional and dilatational parts, namely $\dot{\epsilon}^{p D}$ and $\dot{\epsilon}^{p M}$, respectively, of the inelastic strain rate $\dot{\sim}_{\sim}^{p}$ are accordingly given by

$$
\begin{aligned}
\dot{\epsilon}^{p D} & =\Lambda \frac{\partial \Phi}{\partial \sigma_{e q}}=2 \Lambda \frac{\tilde{\sigma}_{e q}}{\overline{\sigma_{y}}} \\
\dot{\epsilon}^{p M} & =-\Lambda \frac{\partial \Phi}{\partial p_{m}}=3 q_{1} q_{2} f \Lambda \frac{\sinh \left[-\frac{3}{2} q_{2}\left(\tilde{p}_{m}+\tilde{p}_{r}\right)\right]}{\bar{\sigma}_{y}}
\end{aligned}
$$

with $\tilde{\sigma}_{e q}=\frac{\sigma_{e q}}{\overline{\sigma_{y}}}, \tilde{p}_{m}=\frac{p_{m}}{\overline{\sigma_{y}}}$ and $\tilde{p}_{r}=\frac{p_{r}}{\overline{\sigma_{v}}}$, and where $\Lambda$ represents the viscoplastic multiplier.

The evolution law of the isotropic hardening variable $\kappa$ is deduced from the equality of the macroscopic plastic work rate with the microscopic one, see Gurson [1]:

$$
\dot{\kappa}=\frac{\sigma_{e q} \dot{\epsilon}^{p D}-p_{m} \dot{\epsilon}^{p M}}{(1-f) \overline{\sigma_{y}}}
$$

According to Longère et al. [10], see also Longère and Dragon [27], adiabatic heating under dynamic dissipative evolution is evaluated from

$$
\rho C \dot{T}=\sigma_{e q} \dot{\epsilon}^{p D}-p_{m} \dot{\epsilon}^{p M}-r \dot{\kappa}
$$

where $\rho$ and $C$ represent the mass density and specific heat, respectively. The porosity rate $\dot{f}$ is decomposed into a contribution due to growth of existing defects, namely $\dot{f}_{g}$, and a contribution due to the formation of new defects, namely $\dot{f}_{n}$ :

$$
\dot{f}=\dot{f}_{g}+\dot{f}_{n}
$$




$$
\begin{aligned}
& \dot{f}_{g}=(1-f) \underset{\sim}{\operatorname{Tr}} d^{p}=(1-f) \dot{\epsilon}^{p M} \\
& \dot{f}_{g}(0)=f_{0} \\
& \dot{f}_{n}=B \dot{\sigma}_{y} \\
& \dot{f}_{n}(0)=0
\end{aligned}
$$

where $B$ is a function defined in [10].

\subsection{Numerical procedure}

The material behaviour outlined in the previous subsection was implemented as user material (umat) in the engineering finite element computation code Abaqus. The numerical integration is achieved using the classical return mapping procedure combined with the Newton-Raphson solving algorithm, see Aravas [28] and Vadillo et al. [29] for further details. Time increments being small, the elastic tangent operator is used. Dividing Eq. (25) by Eq. (26), or inversely, allows for eliminating the viscoplastic multiplier and defining from the incremental viewpoint:

$$
\Xi_{\Delta}=\Delta \epsilon^{p D} \frac{\partial \Phi_{G T N}}{\partial p_{m}}+\Delta \epsilon^{p M} \frac{\partial \Phi_{G T N}}{\partial \sigma_{e q}}=0
$$

The numerical integration consists thus in solving the following system of equations

$$
\begin{aligned}
& \Phi\left(\sigma_{e q}, p_{m} ; H^{\alpha}\right)=0 \\
& \Xi_{\Delta}=0 \\
& p_{m}=p_{m}^{e}+K \Delta \epsilon^{p M} \\
& \sigma_{e q}=\sigma_{e q}^{e}-3 \mu \Delta \epsilon^{p D} \\
& \Delta H=h\left(\Delta \epsilon^{p D}, \Delta \epsilon^{p M}, \sigma_{e q}, p_{m}, H\right)
\end{aligned}
$$

where $\sigma_{e q}^{e}$ and $p_{m}^{e}$ represent the trial, equivalent stress and pressure, respectively, and $\Delta H$ the system of complementary laws Eqs. (25), (26) and Eqs. (29)-(31) written in the incremental form. The constants $K$ and $\mu$ represent the bulk and shear moduli, respectively. Adiabatic conditions are assumed for strain rates greater than $1 s^{-1}$.

\section{FE enrichment}

The X-FEM approximation adopted in the present work is detailed in the following.

\subsection{Principle}

The eXtended Finite Element Method (X-FEM) belongs to methods aiming at enlarging the scale of spatial discretisation. In particular, X-FEM consists in embedding strong discontinuities, induced by e.g., cracks, inside finite elements by enriching the regular displacement field. The singular functions attempt to reproduce the kinematic consequences of the discontinuities at stake. The current displacement field $u(x, t)$ is generally expressed as:

$$
\left.u(x, t)=\sum_{i \in I} u_{i}(t) N_{i}(x)+\sum_{j \in J} b_{j}(t) N_{j}(x) H(x)+\sum_{k \in K} N_{k}(x) \quad \sum_{l=1}^{4} c_{k}^{l}(t) F_{l}(x)\right)
$$

where $u_{i}(t)$ represents the regular nodal displacement of node $i, b_{j}(t)$ the discontinuous nodal displacement magnitude of node $j$ belonging to a crack-crossed finite element, and $c_{k}^{l}(t)$ the singular nodal displacement magnitude of node $k$ belonging to a crack tip-containing finite element. $N_{i}(x)$ represent the shape functions, $F_{l}(x)$ the four singular functions at the crack tip, and $H(x)$ the generalised Heaviside function with 
$H(x)=\operatorname{sign}\left(d_{\Gamma}(x)\right)$ where $d_{\Gamma}(x)$ denotes a signed distance function from the crack $(H(x)$ takes the value +1 for the nodes located above the crack and -1 for the nodes located below the crack). The total set of nodes is denoted as $I$, the set of nodes belonging to crack-crossed finite elements as $J$, and the set of nodes belonging to crack tip-containing finite elements as $K$, see Fig. 1.

In the case where the crack tip is contained within the finite element, the displacement field expression is

$$
\left.u(x, t)=\sum_{i \in I} u_{i}(t) N_{i}(x)+\sum_{k \in K} N_{k}(x) \sum_{l=1}^{4} c_{k}^{l}(t) F_{l}(x)\right)
$$

According to linear fracture mechanics, in the case of a two-dimensional (2D) elastic problem, the singular functions $F_{l}(x)$ are expressed, in a cylindrical frame $(r, \theta)$, as:

$$
F_{l=1,2,3,4}(r, \theta)=\left[\sqrt{r} \sin \frac{\theta}{2}, \sqrt{r} \cos \frac{\theta}{2}, \sqrt{r} \sin \theta \sin \frac{\theta}{2}, \sqrt{r} \sin \theta \cos \frac{\theta}{2}\right]
$$

The expression above represents a double singularity: (i) the singularity of the displacement field on both sides of the crack due to the creation of free surfaces and (ii) the singularity of the stress field at the crack tip.

In the case where the crack has entirely crossed the finite element, the displacement field expression reduces to

$$
u(x, t)=\sum_{i \in I} u_{i}(t) N_{i}(x)+\sum_{j \in J} b_{j}(t) N_{j}(x) H(x)
$$

\subsection{Adopted approach}

The X-FE method is particularly efficient when applied to 2D problems involving elastic-brittle materials for which the singular functions $F_{l}(x)$ can be determined analytically, see Eq. (40). In ductile materials exhibiting a strongly non linear response, though the formation of a plastic process zone at the crack tip regularises the stress field, the analytical expression of the field in question is however generally not known $a$ priori. On the other hand, choices have to be made concerning the numerical integration, and in particular the spatial distribution of the integration points, as well as concerning the post-representation of the crack.

\subsubsection{Enrichment functions}

As mentioned above, the use of strongly non linear material response makes difficult the analytical determination of the displacement functions at the crack tip. In some cases, the functions at stake may be determined by analytical studies considering simplified material behaviours or by numerical simulations, see Elguedj et al. [24]. The fact that the material of the present study is subject to strain hardening, thermal softening, viscoplasticity and ductile damage, see Section 2, increases significantly the complexity of the functions to be identified.

To overcome this difficulty, an approach consists in using the singular functions known in the elastic context, see e.g., Prabel et al. [30]. This technique allows to reproduce the case where the crack tip is contained within the

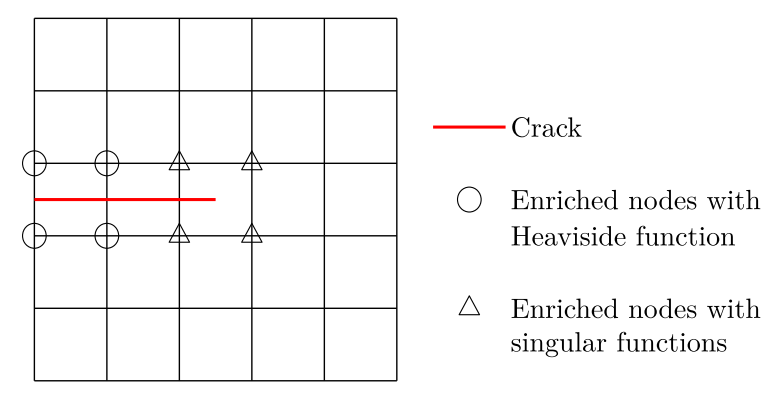

Fig. 1. Principle of designation of enriched nodes. 
element but the evaluation of the stress-strain fields around the crack tip is not correct. The question of the pertinence of such an enrichment is consequently asked. Is thus the use of the Heaviside function only, i.e., without any other enrichment functions, not sufficient - even though the Heaviside enrichment cannot represent the case where the crack is arrested inside the element? Fig. 2 shows the evolution of the equivalent stress (averaged over a patch near the crack tip, like in [17]) for a notched plate consisted of the material behaviour presented in Section 2 and submitted to a tension loading. Curves are drawn for numerical simulations considering i) elastic enrichment functions and ii) Heaviside enrichment functions, for two mesh sizes. According to Fig. 2, only slight discrepancies can be noticed between both approximations. Moreover, using the Heaviside enrichment instead of the elastic enrichment functions allows to reduce drastically the required number of degrees of freedom (dof), 48 dof with the singular functions vs. 16 dof with the sole Heaviside function.

In absence of knowledge of the kinematic field for the complex material behaviour considered in the present work, and according to the aforementioned remarks, we are here using the reduced current displacement field

$$
u(x, t)=\sum_{i \in I} u_{i}(t) N_{i}(x)+\sum_{j \in J} b_{j}(t) N_{j}(x) H(x)
$$

\subsubsection{Spatial distribution of the integration points}

There exist several techniques devoted to capture the crack propagation. The original method consists in subdividing the finite element in which the crack is currently propagating into adaptive sub-triangles, see Moes et al. [14] and an example in Fig. 3. This method however implies re-projecting the state variables values

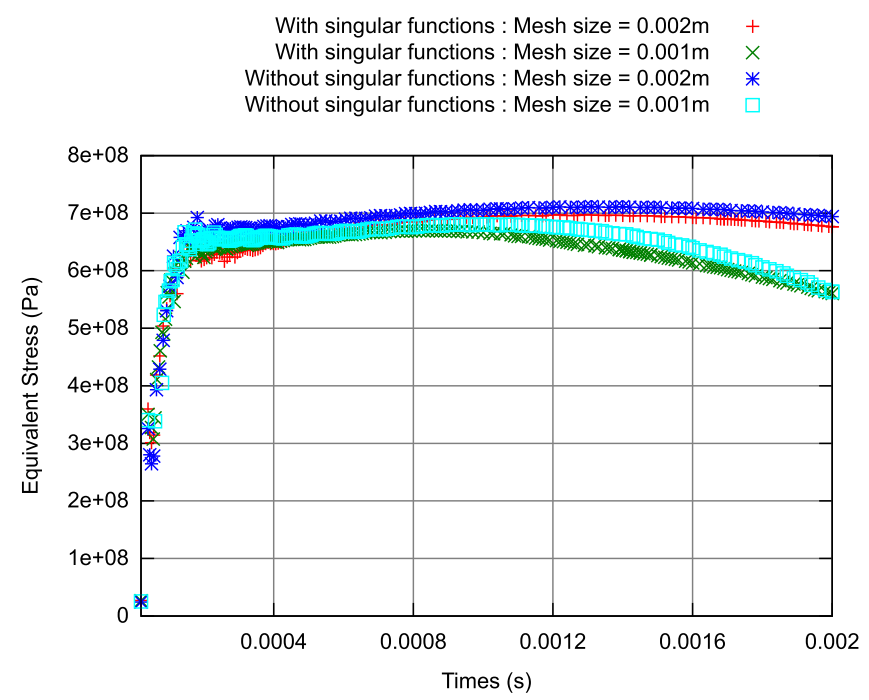

Fig. 2. History of the average equivalent stress with and without singular functions. Case of a notched plate submitted to mode I loading. No crack propagation.

(a) First increment

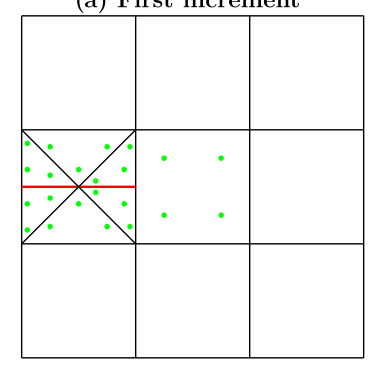

(b) Second increment

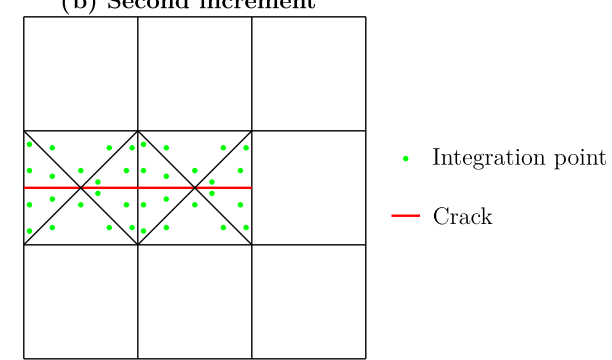

Fig. 3. Gauss Points evolution in finite elements during a crack growth. 
defined at the integration points of the original finite element onto the integration points of the sub-finite elements. The procedure which is easy when dealing with linear evolutions (as it is the case for elastic materials) is far from being trivial when dealing with history- and loading path-dependent, non-linear evolutions (it is the case for the ductile materials considered in the present work). For this reason, the technique consisting in increasing significantly the number of integration points, as suggested by Elguedj et al. [24], of the original finite element has been preferred in the present case. We are here using 64 integration points in enriched elements.

\subsubsection{Issue concerning the nodes belonging to cut and uncut finite elements}

As proposed by $\mathrm{Zi}$ et Belytschko [31], to ensure that the enrichment vanishes in all elements not cut by the crack, the current displacement field $u(x, t)$ eventually takes the form

$$
u(x, t)=\sum_{i \in I} u_{i}(t) N_{i}(x)+\sum_{j \in J} b_{j}(t) N_{j}(x)(H(x)-H(j))
$$

where $H(j)$ corresponds to the global discontinuous step function at node $j$.

\subsubsection{Representation of the crack}

The description and updating of the crack during propagation is achieved using level-set functions, see Moes et al. [32]. The crack is accordingly described as a succession of segments, each one representing a crack growth increment. Segments are described using three functions $(\phi 1, \psi, \phi 2)$ allowing to define, respectively, the old crack tip, the new crack surface increment and the new crack tip. In the case of very small displacement, the level-sets are computed on a fixed grid. When large displacements are involved (with ductile materials) the distortion of the mesh can no longer be neglected, and the grid where the level-sets are computed must evolve. In the present case, the grid is accordingly updated considering large displacement.

\section{Coupling X-FEM and ductile damage}

In the present approach, material and kinematical consequences of the damage process are described via Gurson model within the standard finite element formulation, whereas kinematical consequences of the crack formation (incipience and propagation) are described within the eXtended finite element formulation.

As mentioned in the Introduction, combining a strongly non linear material behaviour involving possible softening and the eXtended Finite Element Method requires a specific strategy allowing for passing from continuous damage mechanics to (non linear) fracture mechanics. On the other hand, due to the lack of time resolved experimental data during the transition between diffuse damage and crack formation, hypotheses regarding the microscopic underlying mechanisms leading to crack initiation, as well as simplifications for the numerical treatment of the crack propagation, have to be made. In particular, because of the complexity of the physical process leading to crack formation, we are here tentatively assuming that the transition between the stages of void coalescence and crack germination is instantaneous. In addition, in order to attenuate the mesh size- and orientation-dependence of the numerical results, we are considering quantities averaged over a patch located at the crack tip and covering several elements, see Fig. 4. The method adopted in the present work is detailed in the sequel.

\subsection{Crack growth orientation $\theta$}

Identifying the crack growth orientation constitutes a challenge in the numerical treatment of crack propagation, in particular when dealing with elastic-(visco) plastic materials. Relying upon linear fracture mechanics results, Haboussa et al. [17] propose transforming a stress intensity factor related criterion (suitable for elastic materials) into an averaged stress related criterion (to be applied to their hardening elastic-plastic material). As mentioned above, this criterion cannot apply to softening materials considered in the present study. In the line of the works by Huespe et al. [25] and Sanchez et al. [33], we are assuming that the crack formation results from a deformation localisation process, notably induced by void coalescence, and that crack orientation may accordingly be deduced from the bifurcation analysis (in the sense of [34], see also Kumar and Drathi 


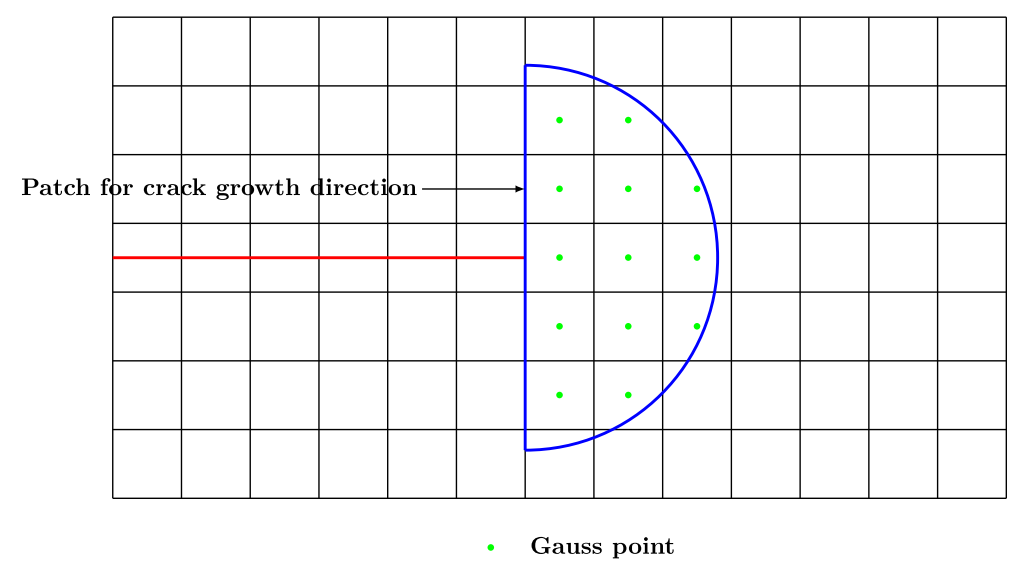

Fig. 4. Shape of the patch used for the evaluation of the crack growth direction.

[35]). According to the bifurcation analysis, see e.g., Yamamoto (1978) and Besson et al. [12], the aim is to find the strain localisation plane defined by its normal $\underline{g}$ satisfying

$$
\operatorname{det}\left(\underline{g}^{t} \underset{\sim}{\sim} \underline{g}\right)=0
$$

where $L$ represents the elastic-plastic tangent operator fourth order tensor. The quantity $\underset{\sim}{A}=\underline{g}^{t} \underset{\approx}{\underline{g}}$ is usually called ãcoustic tensor. The elastic-plastic tangent operator is expressed by

$$
\underset{\sim}{\dot{\sigma}}=\underset{\approx}{L}: \underset{\sim}{\dot{\epsilon}}
$$

From the numerical viewpoint, the principle consists in searching for the normal $g$ rending the quantity $\operatorname{det}(\underset{\sim}{A})$ slightly negative.

Preventing the value of $\operatorname{det}(A)$ to become negative or zero, viscosity (viscoplasticity in the present case) thus does not allow to obtain the bifurcation condition and acts accordingly as a 'regularising' parameter. To nevertheless apply the bifurcation analysis for identifying the crack propagation plane, we are freezing the material state, so the GTN yield locus expressed in Section 2 in the form $\Phi(\underset{\sim}{\sigma}, \kappa, \dot{\kappa}, T, f)$ becomes $\Phi_{*}(\underset{\sim}{\sigma}, \kappa, T, f)=$ $\Phi\left(\underset{\sim}{\sigma}, \kappa, \dot{\kappa}_{n}, T, f\right)$ for the bifurcation analysis. The strain rate induced overstress $\sigma_{v p}\left(\dot{\kappa}_{n}, T_{n}, \ldots\right)$ is consequently viewed as a constant contribution to the yield stress, as is the initial radius $R_{0}$.

The modified GTN plastic potential in the frozen state $\Phi_{*}$ is expressed as

$$
\Phi_{*}(\underset{\sim}{\sigma}, \kappa, T, f)=\left(\frac{\sigma_{e q}}{\overline{\sigma_{y}}}\right)^{2}+2 q_{1} f \cosh \left(-\frac{3}{2} q_{2} \frac{p_{m}+p_{r}}{\overline{\sigma_{y}}}\right)-\left(1+q_{3} f^{2}\right)
$$

where $\bar{\sigma}_{y}$ * represents the yield stress in the frozen state, with a rate independent yield stress part $\left(\sigma_{y}\right)$ similar to Eq. (21) and a rate dependent part $\left(\sigma_{y_{v p}}^{*}\right)$ which is viewed as a constant contribution:

$$
{\overline{\sigma_{y}}}^{*}(\kappa, T)=\sigma_{y}(\kappa, T)+\sigma_{y_{v p}}^{*}
$$

The elastic-plastic tangent operator $\underset{\approx}{L}$ in Eq. (45) is expressed as

$$
\underset{\approx}{\sim} \underset{\approx}{C}-\frac{(\underset{\approx}{C: \underset{\sim}{m})} \otimes(\underset{\sim}{m}: \underset{\approx}{C})}{\Omega}
$$

with $\underset{\sim}{m}$ and $\Omega$ given by

$$
\underset{\sim}{m}=\frac{\partial \Phi_{*}}{\partial \underset{\sim}{\sigma}}
$$




$$
\Omega=\underset{\sim}{m}: \underset{\approx}{C}: \underset{\sim}{m}-\frac{\partial \Phi_{*}}{\partial f}(1-f) \operatorname{Tr}[\underset{\sim}{m}]-(\underset{\sim}{\sigma}: \underset{\sim}{m}) \zeta
$$

The reader can refer to Appendix for further details on the application of the bifurcation analysis.

To evaluate the crack growth orientation $\theta$, we are using a patch covering several finite elements near the crack tip, see Fig. 4. For each integration point located within the patch satisfying the bifurcation condition, two localisation planes are typically found. Fig. 5 shows an example of localisation planes distribution for every integration points located within the patch, with the green data corresponding with one solution of the bifurcation analysis and the red data with the other one. In order to select the localisation plane among both potential ones, we are averaging the plane directions over the patch then we are computing an averaged plastic strain tensor $\tilde{\epsilon}_{i j}^{p}$ defined by

$$
\tilde{\epsilon}_{i j}^{p}=\frac{\int_{H D i s k} e^{-\alpha \frac{d^{2}}{R^{2}}} \epsilon_{i j}^{p}(M) d V_{M}}{\int_{H D i s k} e^{-\alpha \frac{d^{2}}{R^{2}}} d V_{M}}
$$

where $\epsilon_{\sim}^{p}$ represents the plastic strain tensor at the integration point $M, d$ the distance between the point $M$ and the crack tip, $R$ the patch radius and $\alpha$ a constant $(\alpha=10)$. The averaged plastic strain tensor $\tilde{\epsilon}_{i j}^{p}$ is then projected onto both localisation planes to form an opening strain $\epsilon_{g g}$ and a shear strain $\gamma_{g n}$ according to

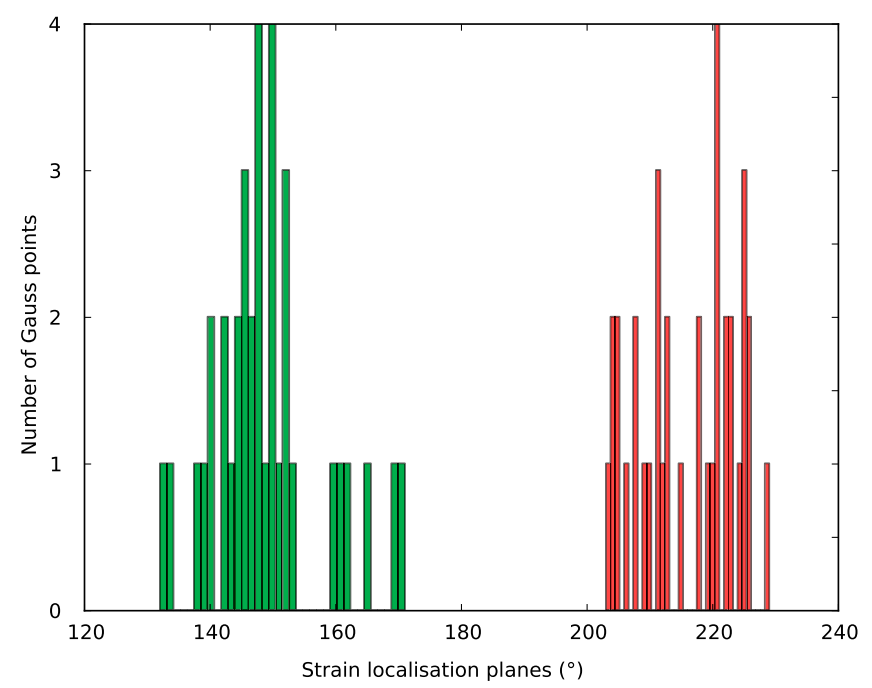

Fig. 5. Example of distribution of the localisation planes.

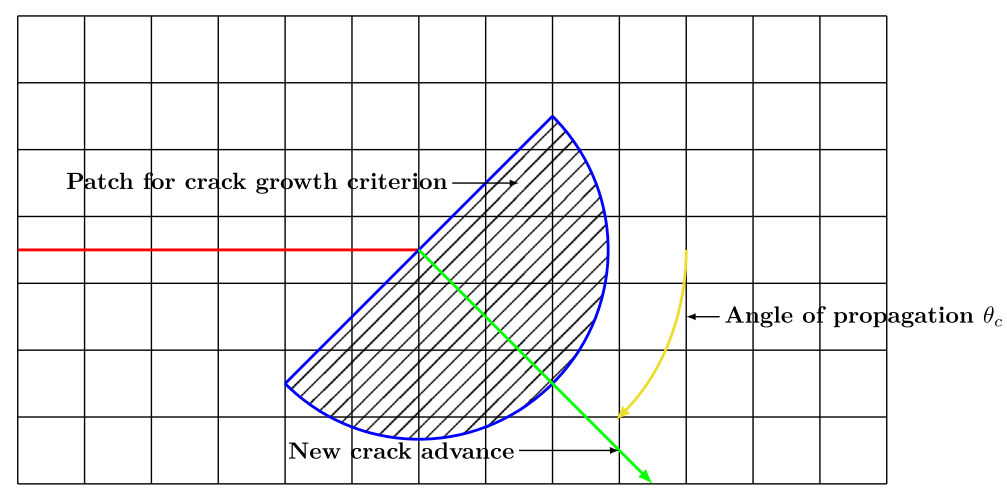

Fig. 6. Shape of the patch used for the evaluation of the crack growth criterion. 


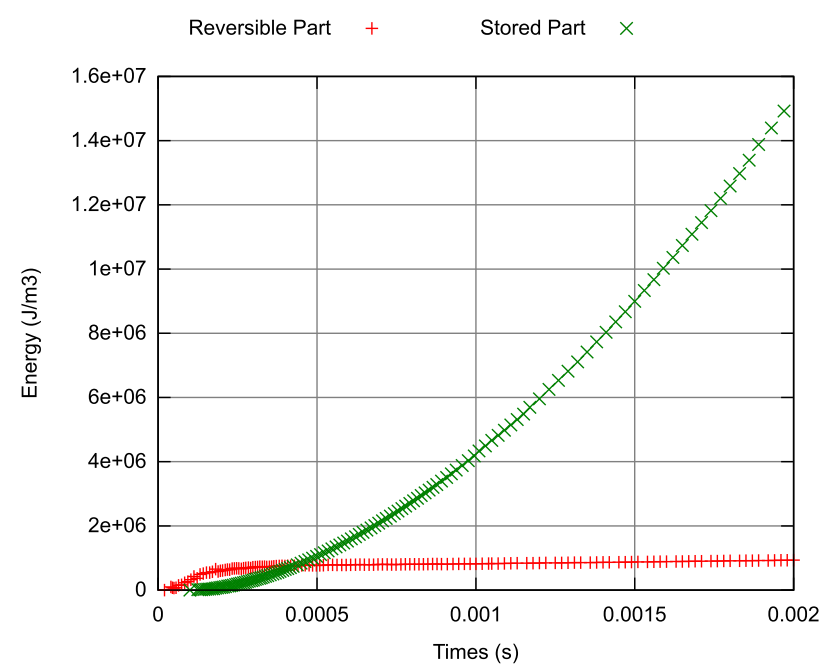

Fig. 7. Evolution of the stored and reversible part of the energy in the case of a plate submit to mode I loading.

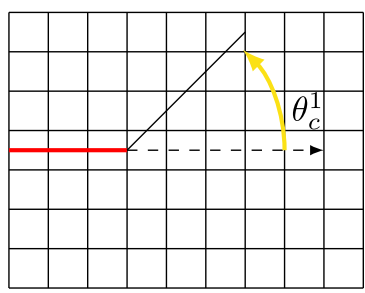

Step 1

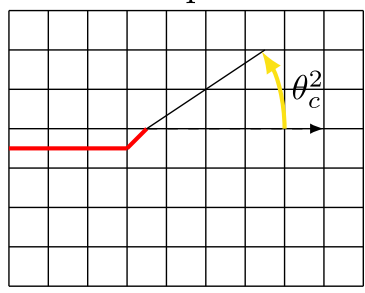

Step 3

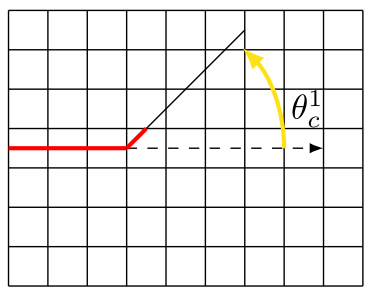

Step 2

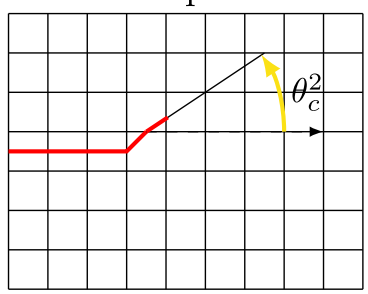

Step 4

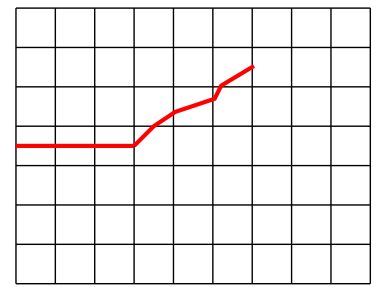

Step $n$

Fig. 8. Exhaustion approach.

$$
\begin{aligned}
& \epsilon_{g g}^{i}=\underline{g_{i}{ }^{t}} \underset{\sim}{\tilde{\epsilon}^{p}} \underline{g_{i}} \\
& \gamma_{g n}^{i}=2\left(\underline{n_{i}{ }^{t}} \underset{\sim}{\tilde{\epsilon}^{p}} \underline{g_{i}}\right)
\end{aligned}
$$




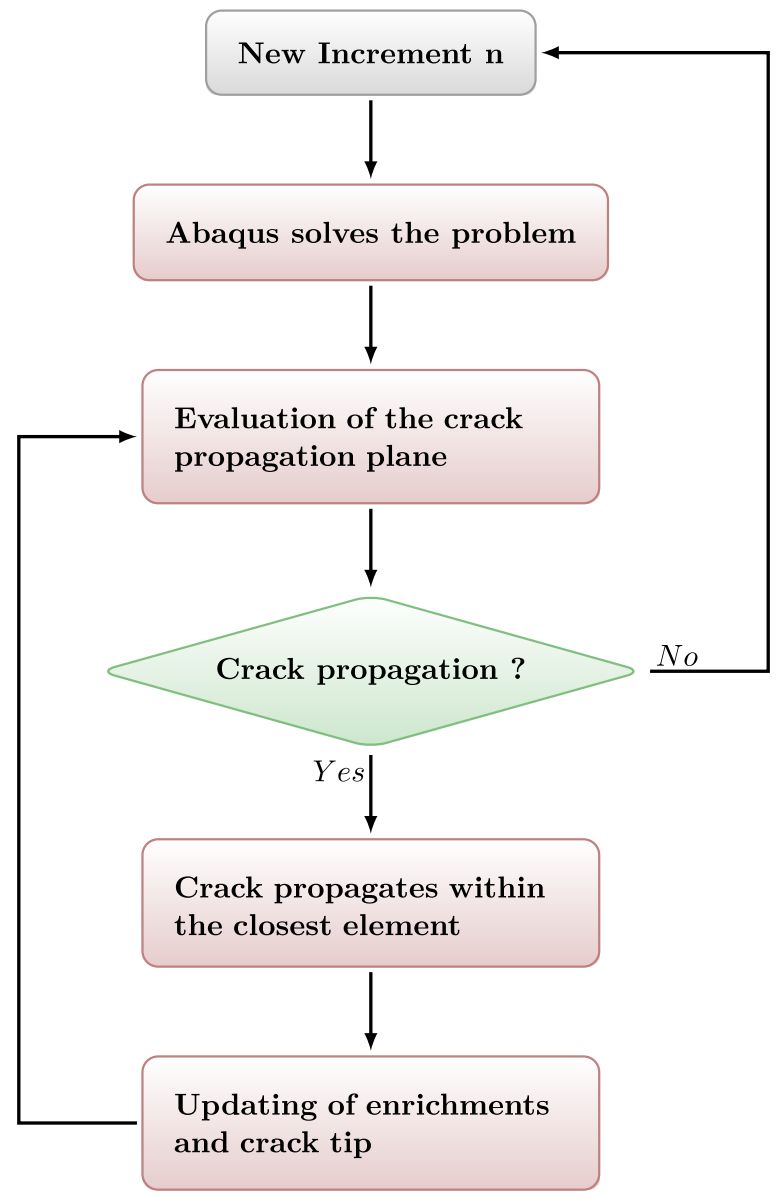

Fig. 9. Algorithm.

where $g_{i}$ and $n_{i}$ represent the vectors, respectively normal and tangential to the localisation plane $i$. We are now considering an equivalent plastic strain $Q_{i}$ expressed as

$$
Q_{i}=\sqrt{\left\langle\epsilon_{g g}^{i}\right\rangle^{2}+\left(\frac{\gamma_{g n}^{i}}{\sqrt{3}}\right)^{2}}
$$

The plane which maximises $Q_{i}$ is chosen as localisation plane and further crack growth plane, of orientation $\theta$. This method is similar to the approach proposed by Kumar and Drathi [35] (see also Rabczuk and Belytschko [36]).

\subsection{Crack growth criterion}

When materials exhibit significant strain softening and high ductility (as it is the case herein), it is no longer possible to use as crack propagation indicators the critical value of the (local) stress intensity factor $\mathrm{K}$ nor the critical value of the (global) Griffith energy release rate $\mathrm{G}$ or Rice-Cherepanov J-Integral. The usual approach consists then in using the critical value of an internal variable, for instance the plastic shear strain or the damage related variable, see e.g., Hambli [37] and Lievers et al. [38]. This approach has however to face up the softening regime induced pathological mesh dependence of the numerical results in the very close vicinity of the crack tip. To reduce this mesh dependence, Haboussa et al. [17] suggest averaging the 


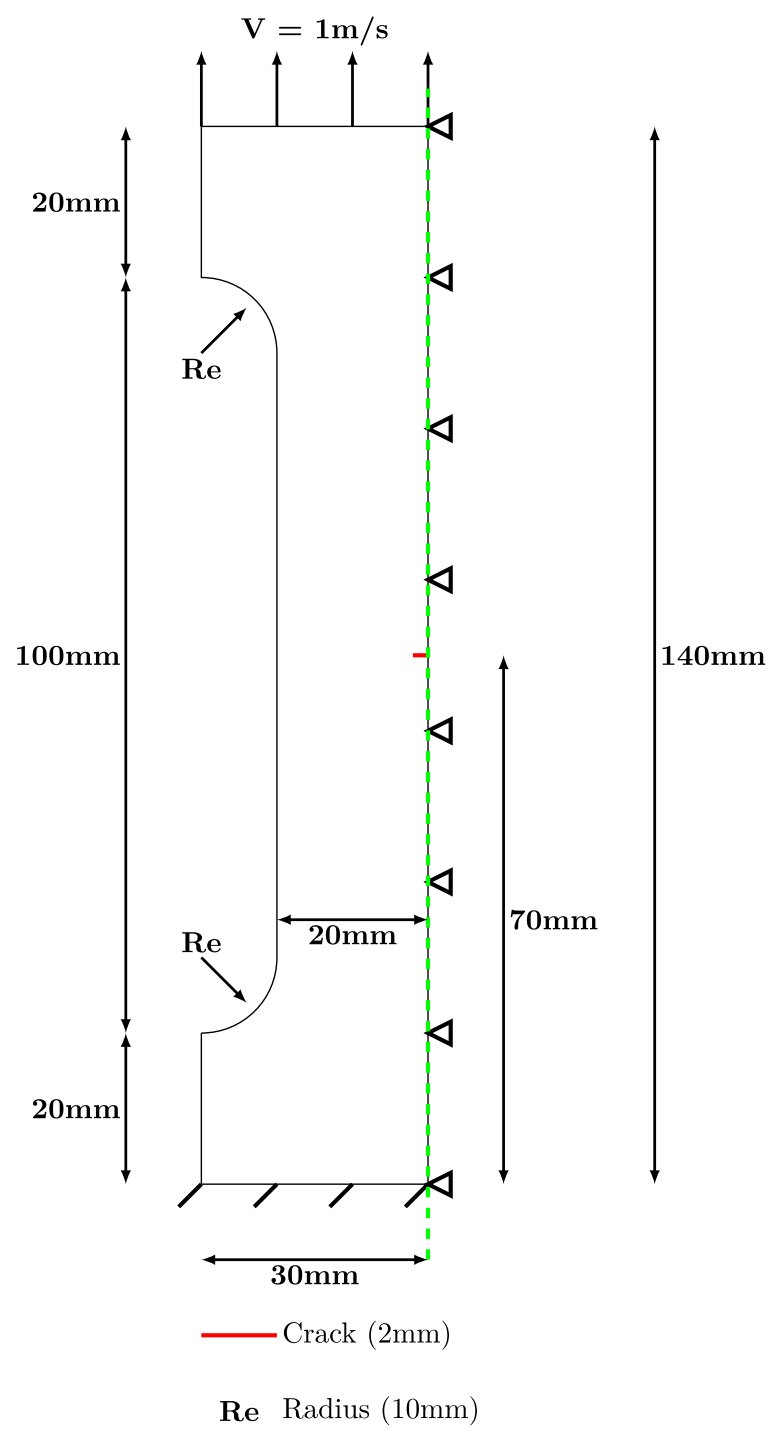

Fig. 10. Crack containing plate under tension loading.

quantities of interest (stress, strain, etc.) over an area (a patch) located at the crack tip. Unlike the aforementioned authors who considered an elastic-plastic material behaviour with positive strain hardening, we are here dealing with elastic-plastic material behaviour exhibiting progressively negative strain hardening. The direct use of a stress (in fact the stress averaged over the patch) related criterion is consequently no longer suitable.

The strain hardening in Eq. (19) describes phenomenologically the microscopic effects of dislocation accumulation and annihilation mechanisms. Starting from a positive hardening material behaviour, the competition between both aforementioned mechanisms may progressively lead to a negative hardening material behaviour and a decrease of the (always positive) slope of the stored energy. We are here assuming that (i) below a critical value of the stored energy, dislocations and void growth related micro-mechanisms are able to accommodate the applied deformation and (ii) from this critical value of the stored energy cracking becomes the only mechanism able to accommodate extra deformation. To describe the transition from diffuse damage to crack formation, a criterion involving a stored energy related quantity seems consequently suitable. 
We are accordingly averaging the stored energy over a half circle shape patch containing a finite number $p$ of elements (according to Eq. (55)), see Fig. 6, located at the crack tip and which symmetry axis is collinear with the crack growth direction, according to

$$
\begin{aligned}
& W_{\text {patch }}=\frac{1}{A} \sum_{i=1}^{p} \omega_{s}^{i} A^{i} \\
& A=\sum_{i=1}^{p} A^{i}
\end{aligned}
$$

where $W_{\text {patch }}$ represents the stored energy averaged over the patch, $A$ and $A^{i}$ the patch area and the element $i$ area, respectively. $\omega_{s}^{i}$ represents the (averaged over all the Gauss points) stored energy of the element $i$. For a given patch area, it is consequently possible to conduct a convergence study regarding the mesh size dependence.

In the present approach, the crack propagates if

$$
F\left(W_{\text {patch }}\right)=1-\frac{W_{c}}{W_{\text {patch }}}>0
$$

In Fig. 7 are drawn the relative contributions of both the averaged, reversible and stored parts of the free energy. It is clearly visible that the stored energy value becomes progressively much greater than the reversible energy one.

\subsection{Crack growth magnitude}

The method retained in the present work consists in propagating the crack from an element to another one as long as the crack growth criterion is satisfied. The methodology is shown in Fig. 8. First we are searching for the crack propagation plane according to the bifurcation analysis (Step 1, Section 4.1). If the crack growth criterion is satisfied, see Eq. (56), the crack propagates within the closest element (Step 2, Section 4.2). For this new crack tip, we restart the same approach (Steps 3 and 4). Finally we obtain the new crack increment when the localisation condition is not satisfied (Step n, see Fig. 8). The algorithm is presented in Fig. 9.

This 'exhaustion' method does not allow to control the crack propagation speed but allows to reproduce potential bifurcation of the crack propagation for large crack advances, i.e., when the crack propagates through several elements during a single time step increment.

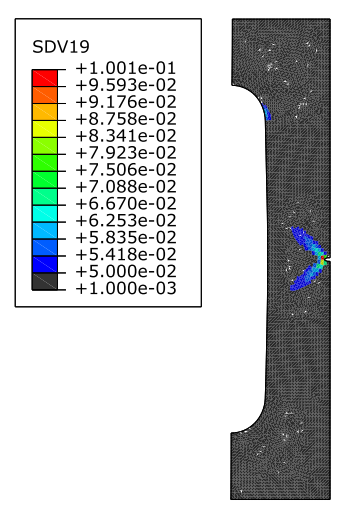

(a)
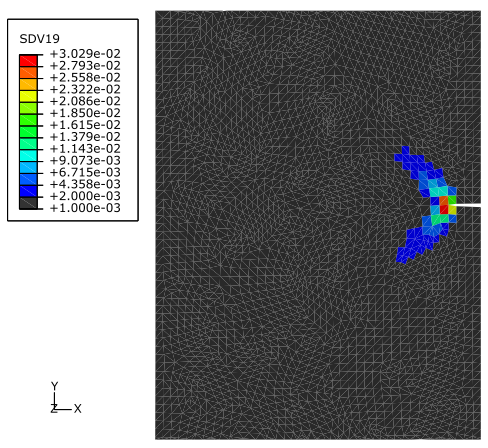

(b)

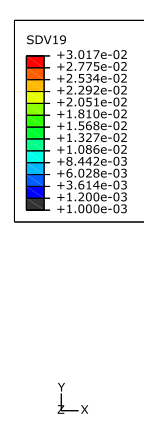

(c)

Fig. 11. (Application 1) Porosity field before the crack propagation starting: (a) Mesh size 1 (coarse), (b) Mesh size 2 (medium), and (c) Mesh size 3 (fine). 


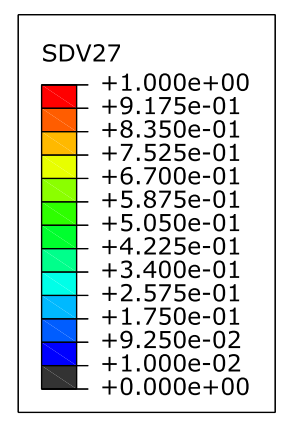

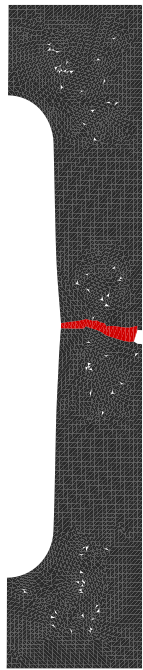

(a)

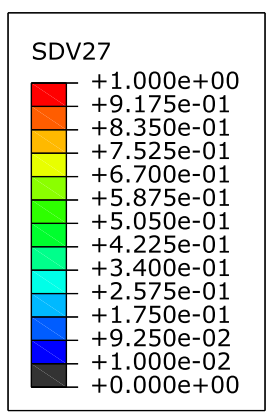

(b)
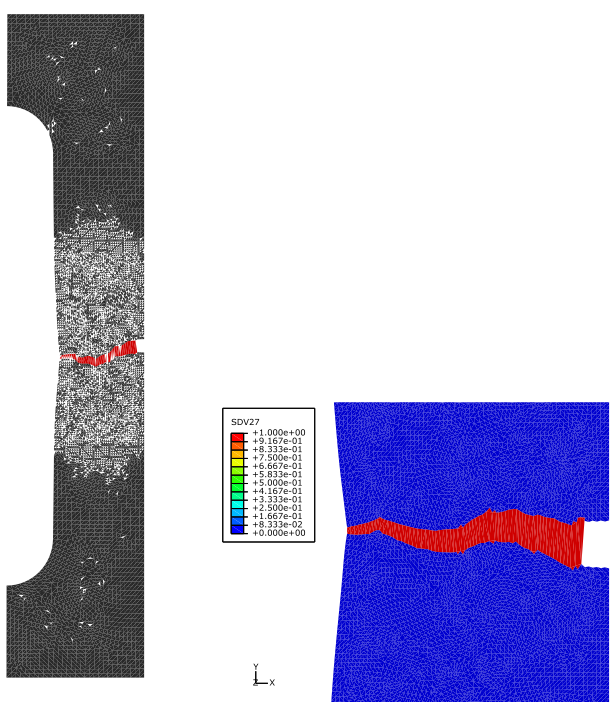

(c)

Fig. 12. (Application 1) Element deletion criterion field for the element deletion based method: (a) Mesh size 1 (coarse), (b) Mesh size 2 (medium), and (c) Mesh size 3 (fine).

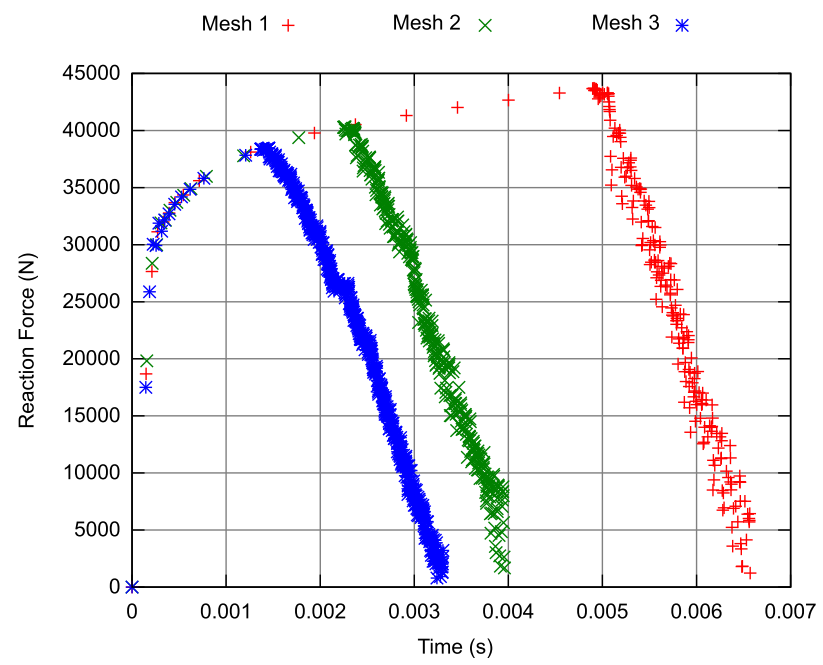

Fig. 13. (Application 1) Evolution of the reaction force using the element deletion based method.

\section{Application}

This section aims at comparing results obtained on one hand from the usual, element deletion based method within the standard FEM formulation and on the other hand from the methodology developed in Section 3 and 4 employing the eXtended FEM, both using the engineering computation code Abaqus. The rate equations of the material behaviour detailed in Section 3.1 are integrated via a user material (umat) subroutine. The numerical time integration scheme is implicit and small strain hypothesis is assumed. For reason of confidentiality, some constant values are not given. 


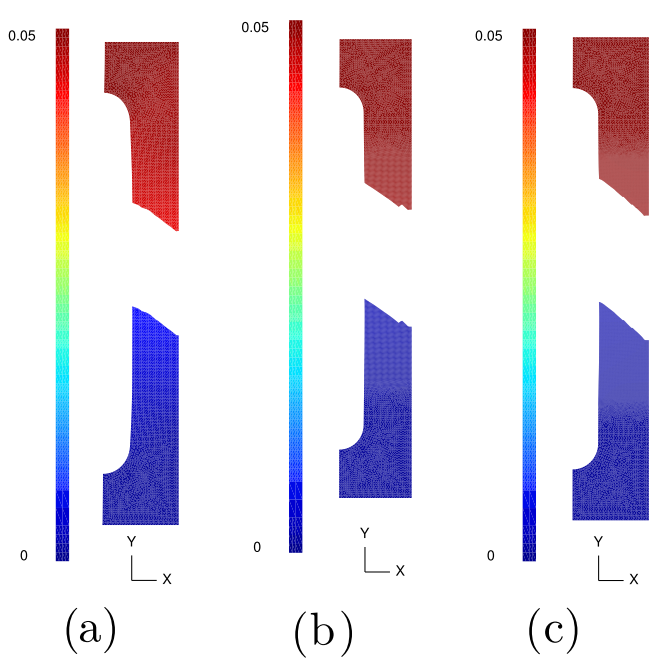

Fig. 14. (Application 1) $Y$-axis displacement field using the proposed method: (a) Mesh size 1 (coarse), (b) Mesh size 2 (medium), and (c) Mesh size 3 (fine).

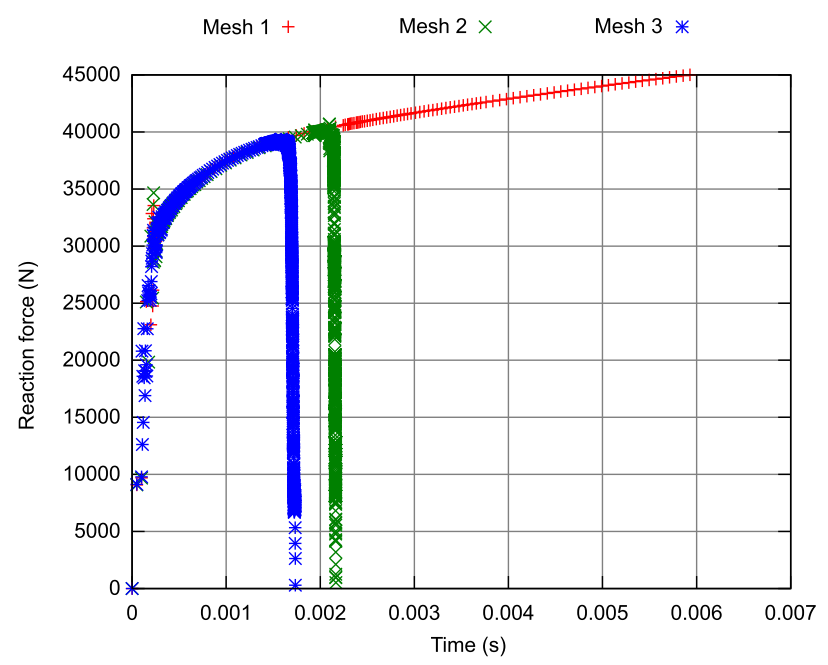

Fig. 15. (Application 1) Evolution of the reaction force using the proposed method.

In the case of the element deletion based model, the plate is meshed using 4 node finite elements with 4 integration points. Elements are deleted as soon as the porosity reaches the (arbitrarily chosen) critical value of 0.1 .

In the case of the model developed in the present work, the plate is meshed using 4 node finite elements with 64 integration points, enriched according to the formulation outlined in Section 3.2 via a user element (uel) subroutine. The propagation method requires two constant values, respectively linked to the stored energy and to the patch radius. For the present evaluation of the methodology, the values of the quantities at stake have been arbitrarily fixed: $W_{c}=4 \mathrm{MJ} / \mathrm{m}^{3}$ and $R=5 \mathrm{~mm}$.

\subsection{Application 1: crack containing plate}

In this first application, we are considering a $2 \mathrm{D}$ (plane strain, thickness $=4 \mathrm{~mm}$ ), crack containing plate made of a ductile material, namely the mild steel used in [39], see Fig. 10. According to the plate symmetry, half of the plate is meshed. The upper side of the plate is submitted to a vertical velocity of $1 \mathrm{~m} / \mathrm{s}$ and the lower 


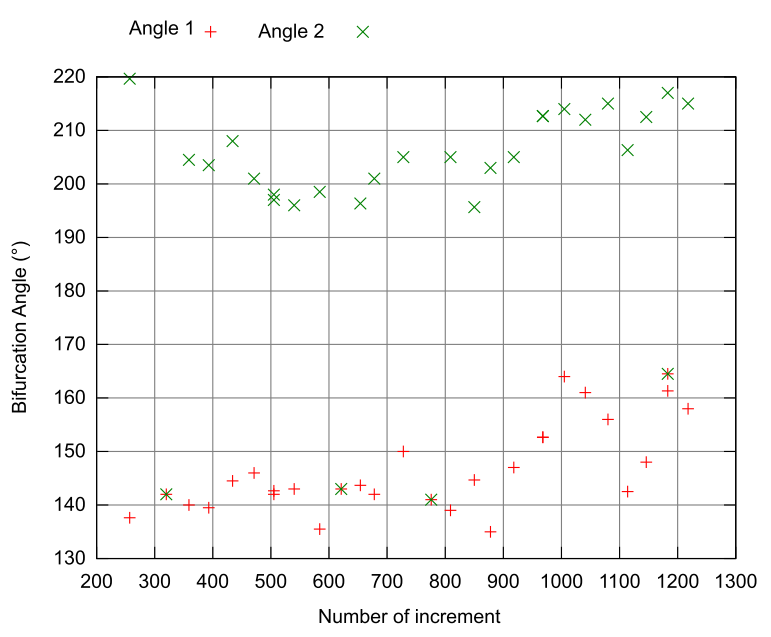

(a)

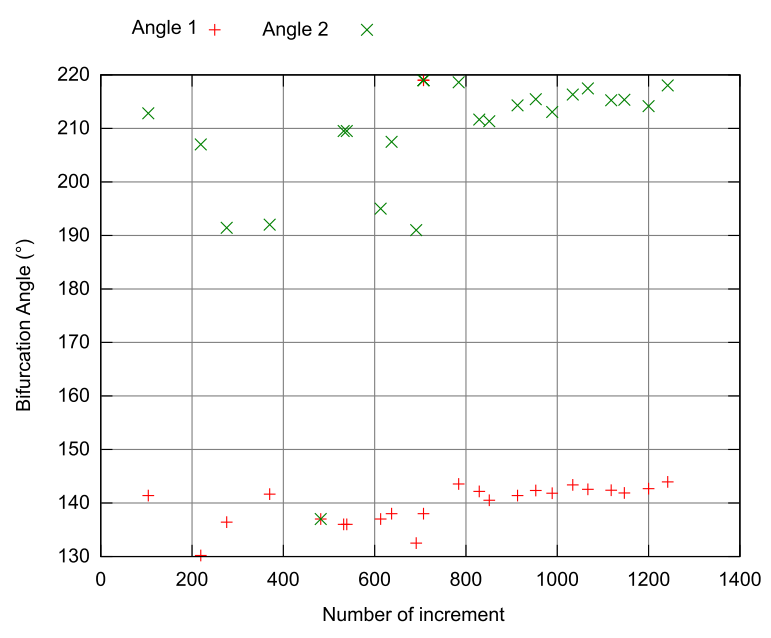

(b)

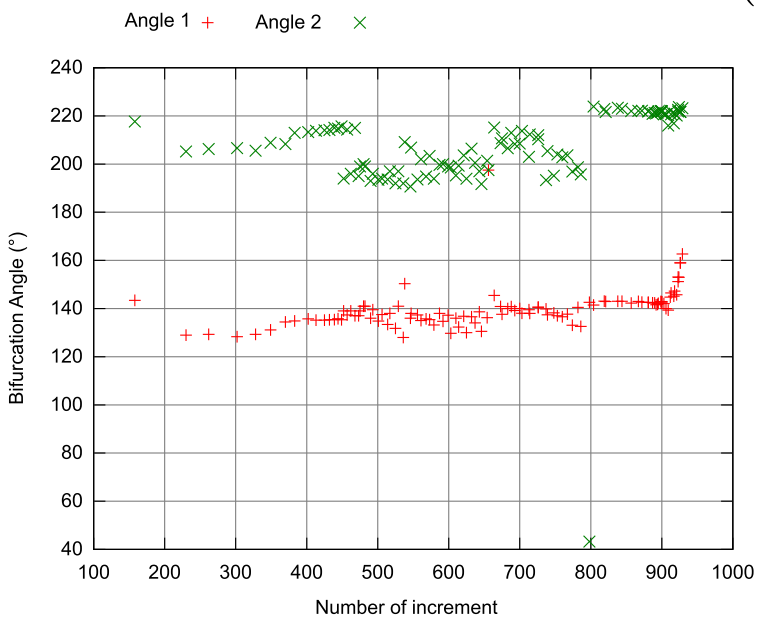

(c)

Fig. 16. (Application 1) Bifurcation angle using the proposed approach: (a) Mesh size 1 (coarse), (b) Mesh size 2 (medium), and (c) Mesh size 3 (fine).

side is clamped. Three mesh sizes have been used for each method in order to estimate the mesh size dependence, with the mesh size 1 corresponding with the coarsest and the mesh size 3 with the finest.

Fig. 11 shows the porosity map just before the crack propagation for every mesh size. According to this figure, it is clearly visible that the porosity value is maximum for the element located at the crack tip and that there are two symmetric (with respect to $X$-axis), porosity localisation branches initiating from the crack tip and oriented with an angle of about $\pm 35^{\circ}$ whatever is the mesh size.

\subsubsection{Element deletion based method}

In the case of the element deletion based method, the crack propagation is controlled by the maximum value of the porosity. Fig. 12 shows the plate after the whole propagation of the numerical crack throughout its width. One can see on this figure that depending on the mesh size, the crack initially propagates toward the lower or the upper plate side. One can also note that the crack eventually tends to propagate horizontally, i.e., in the direction of the maximum porosity value as expected, whatever is the mesh size. 


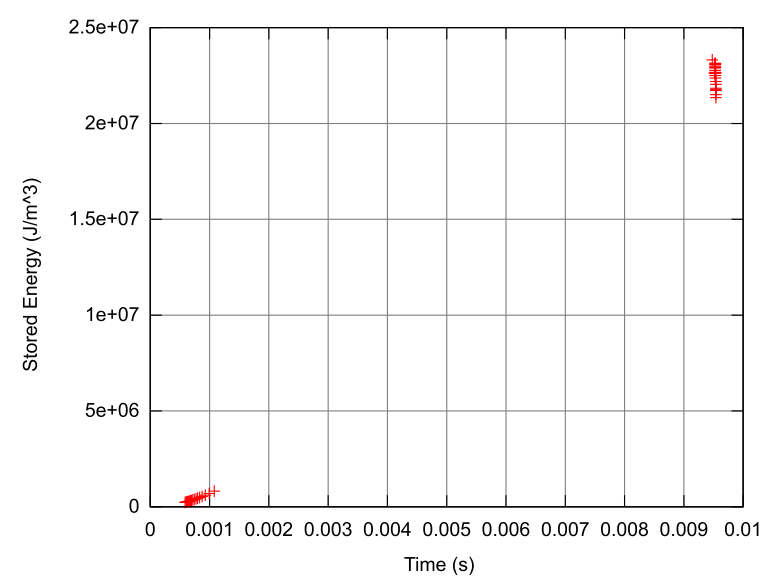

(a)

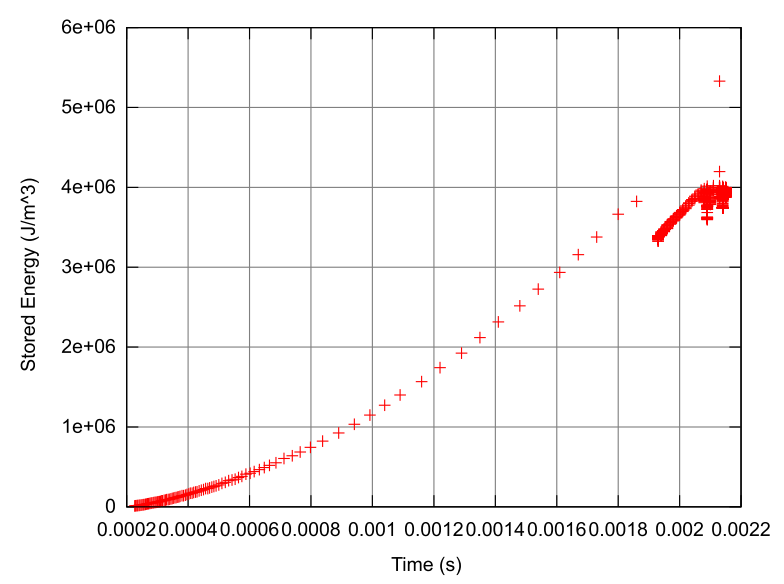

(b)

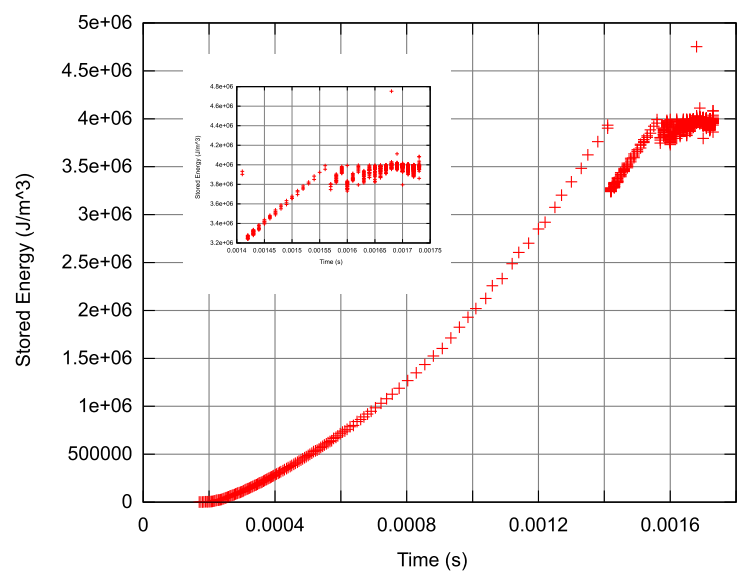

(c)

Fig. 17. (Application 1) Evolution of the stored energy using the proposed approach: (a) Mesh size 1 (coarse), (b) Mesh size 2 (medium), and (c) Mesh size 3 (fine).

The reaction force is drawn for every mesh size in Fig. 13. The latter shows that the time at crack initiation (starting of the drop in the reaction force) is strongly dependent on the mesh size and that the duration for the reaction force to become null is relatively long.

\subsubsection{Proposed method}

According to the bifurcation analysis, the crack propagation is expected to follow one of the two localisation directions mentioned previously. This is confirmed in Fig. 14 where one can observe that the crack propagates with an angle of about $135^{\circ}$. The fact that the propagation angle is positive (and not negative) is a purely numerical consequence.

The reaction force is drawn for every mesh size in Fig. 15. One can once again observe a dependence of the time at crack initiation on the mesh size with however a tendency to convergence for the finest mesh sizes. Unlike the progressive drop in the reaction force observed when using the element deletion based method, see Fig. 13, one can note according to Fig. 15 a very fast drop in the reaction force when using the proposed method. 


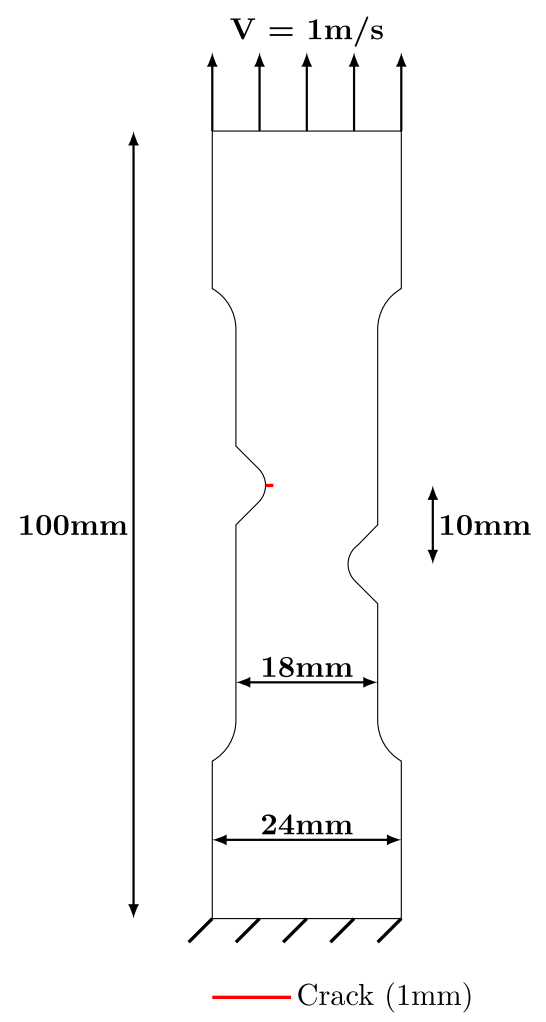

Fig. 18. Asymmetric notched plate under tension loading.

\subsubsection{Comments on the results obtained from the proposed method}

(i) On the angle of propagationFig. 16 shows the two potential crack directions $\theta$ all along the crack propagation according to the bifurcation analysis. Whatever is the mesh size, one can observe that the direction retained, using the criterion expressed via Eq. 54 and corresponding with the red curve in Fig. 16, remains quasi unchanged during the crack growth.

(ii) On the crack initiation

To complete the analysis of the proposed approach, the stored energy $W_{\text {patch }}$ is drawn for the three mesh sizes in Fig. 17.

It must be recalled that the stored energy $W_{\text {patch }}$ (allowing further to check the crack initiation criterion) is computed only if the bifurcation analysis leads to the determination of potential localisation (assumed further as crack propagation) directions. According to Fig. 17.a, one can see that for the coarse mesh size (1) configuration, there exists a long time during which no localisation direction is found (the bifurcation condition not being satisfied). Second, the serration observed in Figs. 17(b) and (c) is a feature of the crack propagation.

\subsection{Application 2: asymmetrically notched plate}

In this second application we are considering a $2 \mathrm{D}$ (plane strain, thickness $=2 \mathrm{~mm}$ ), asymmetrically notched plate made of a the same ductile material than previously, see Fig. 18. The upper notch contains a $1 \mathrm{~mm}$-length crack, and the upper side of the plate is submitted to a vertical velocity of $1 \mathrm{~m} / \mathrm{s}$ while the lower side is clamped. Three mesh sizes have also been considered for each model.

Fig. 19 shows the porosity field just before the crack propagation. As expected, the porosity value is once again maximum for the element located at the upper notch crack tip. There are also two symmetric, porosity localisation branches initiating from the upper notch crack tip and heading for the upper branch to the right 


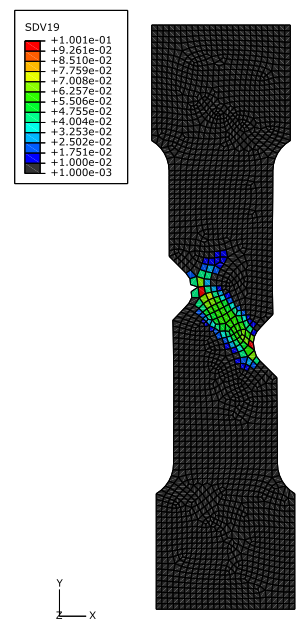

(a)

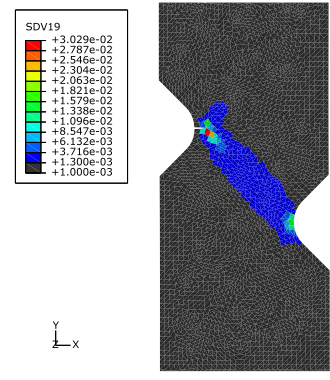

(b)

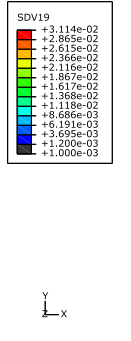

(c)

Fig. 19. (Application 2) Porosity field before the crack propagation starting: (a) Mesh size 1 (coarse), (b) Mesh size 2 (medium), and (c) Mesh size 3 (fine).

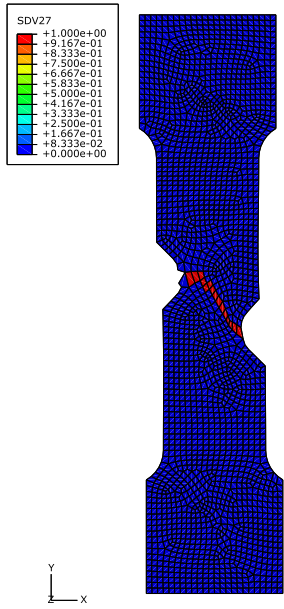

(a)

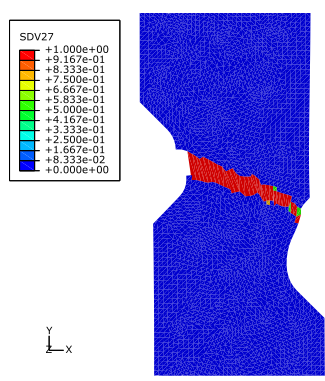

(b)
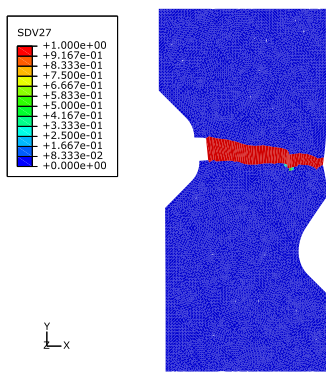

(c)

Fig. 20. (Application 2) Element deletion criterion field for the element deletion based method: (a) Mesh size 1 (coarse), (b) Mesh size 2 (medium), and (c) Mesh size 3 (fine).

specimen edge with an angle of about $+45^{\circ}$, and for the lower branch to the second (lower) notch with an angle of about $-45^{\circ}$.

\subsubsection{Element deletion based method}

According to Fig. 20, one can notice a strong dependence of the crack propagation path on the mesh size using the element deletion based method. For the coarse mesh size configuration (a), the crack starts propagating upwards over some elements then bifurcates downwards toward the lower notch. For the medium mesh size (b), the crack propagates straight toward the lower notch. For the fine mesh size (c), the crack also propagates straight but toward the right specimen edge above the lower notch. 


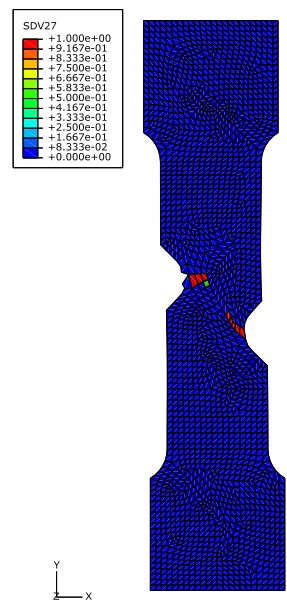

(a)

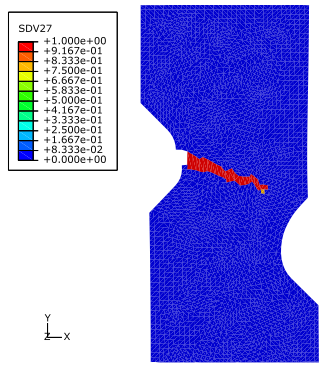

(b)

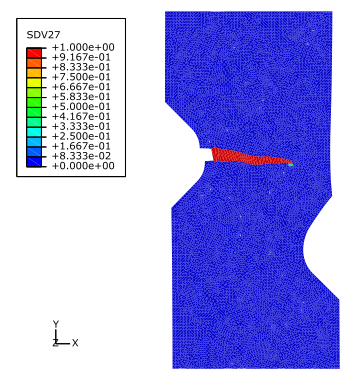

(c)

Fig. 21. (Application 2) Crack path before failure of the plate using the element deletion based method.

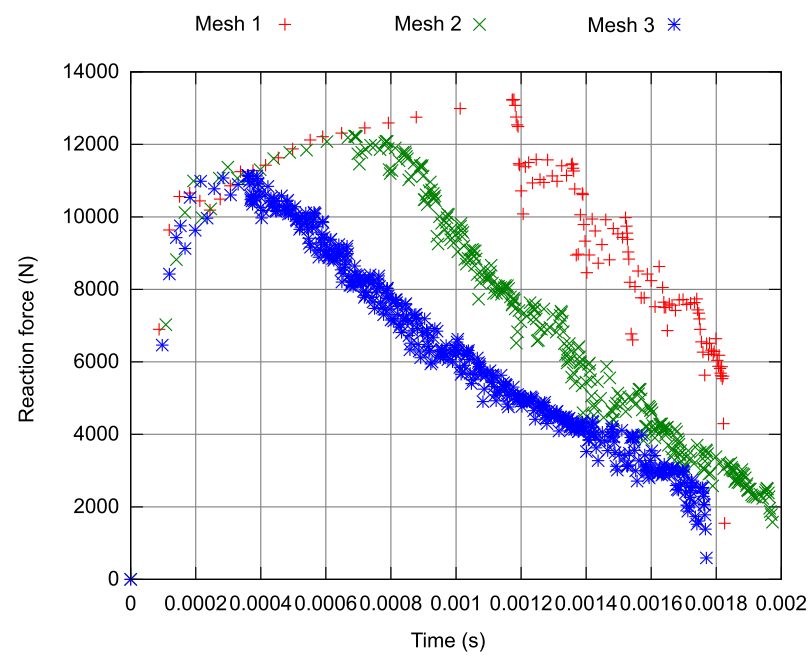

Fig. 22. (Application 2) Evolution of the reaction force using the element deletion based method.

Fig. 21 shows the intermediate crack propagation state in relation with the final state in Fig. 20. One can clearly see that for the coarse mesh size configuration, a second crack initiates from the lower notch. This is not the case when the mesh size becomes finer, see (b) and (c).

The reaction force is drawn for every mesh size in Fig. 22. The latter shows that the time at crack initiation (starting of the drop in reaction force) is also strongly dependent on the mesh size and that the drop in the reaction force is relatively smooth.

\subsubsection{Proposed method}

As mentioned previously, when employing the bifurcation analysis, the crack propagation is expected to follow one of the two localisation directions, as shown in Fig. 23 where one can observe that the crack propagates with an angle of about $-45^{\circ}$ toward the lower notch.

The reaction force is drawn for every mesh size in Fig. 24. A slight dependence of the time at crack initiation on the mesh size can be noticed, as well as a relatively fast drop in the reaction force. 


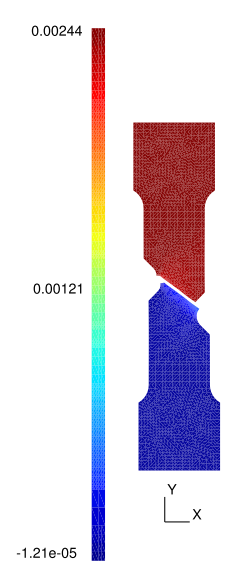

(a)

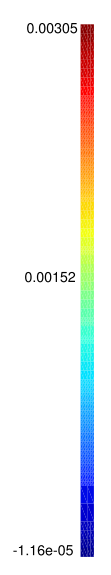

(b)

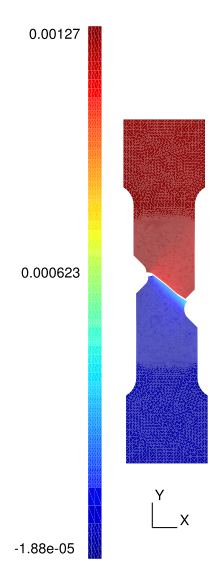

(c)

Fig. 23. (Application 2) Y-axis displacement field using the proposed method: (a) Mesh size 1 (coarse), (b) Mesh size 2 (medium), and (c) Mesh size 3 (fine).

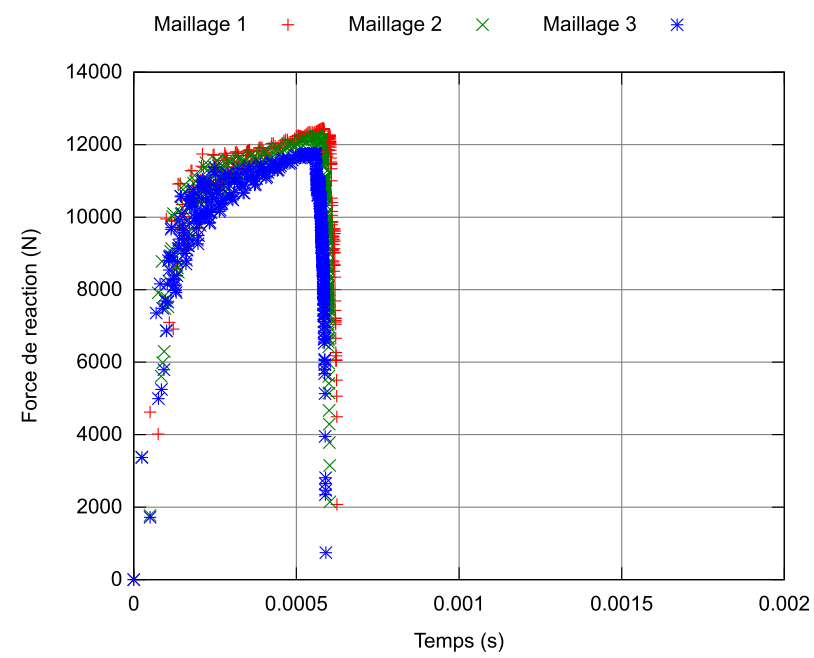

Fig. 24. (Application 2) Evolution of the reaction force using the proposed method.

\subsubsection{Comments on the results obtained from the proposed method}

(i) On the angle of propagationThe two potential crack directions $\theta$ all along the crack propagation obtained from the bifurcation analysis for this second application are drawn in Fig. 25. Whatever is the mesh size, these directions remain unchanged during the crack growth.

(ii) On the crack initiation

The stored energy $W_{\text {patch }}$ is drawn for every mesh size in Fig. 26.

Whatever is the mesh size, the stored energy $W_{\text {patch }}$ increases until reaching the critical value of $W_{c}$ then remains constant.

\subsection{Summary}

As mentioned previously, the crack propagation is controlled by the maximum value of the porosity in the case of the element deletion based method whereas it is controlled by the damage induced deformation localisation in the case of the methodology proposed in the present paper. 


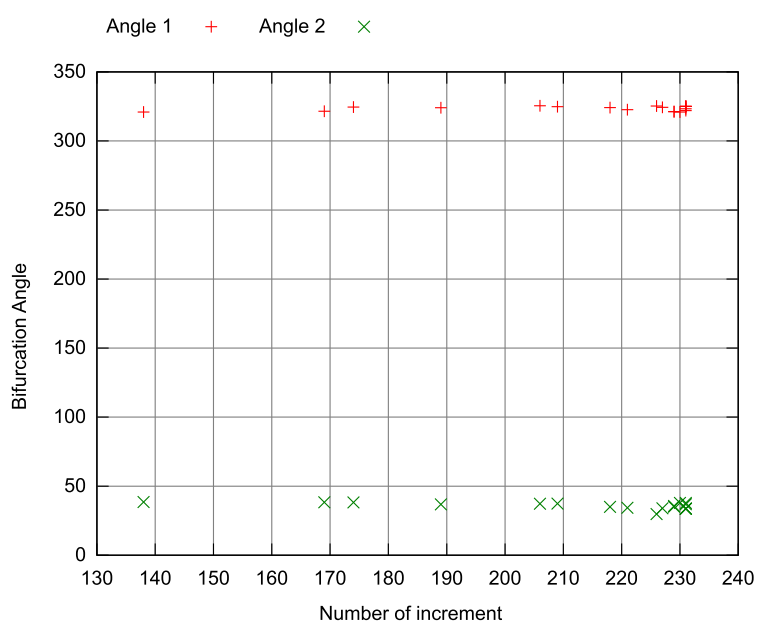

(a)

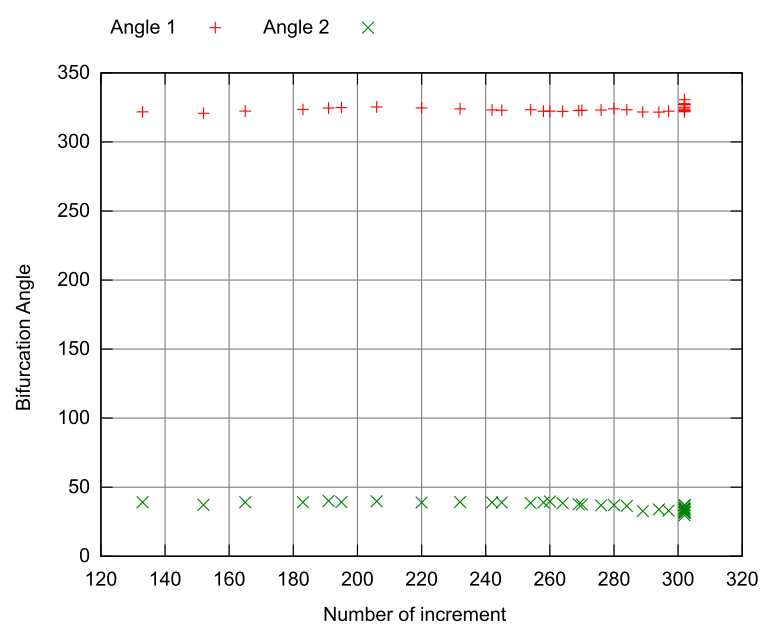

(b)

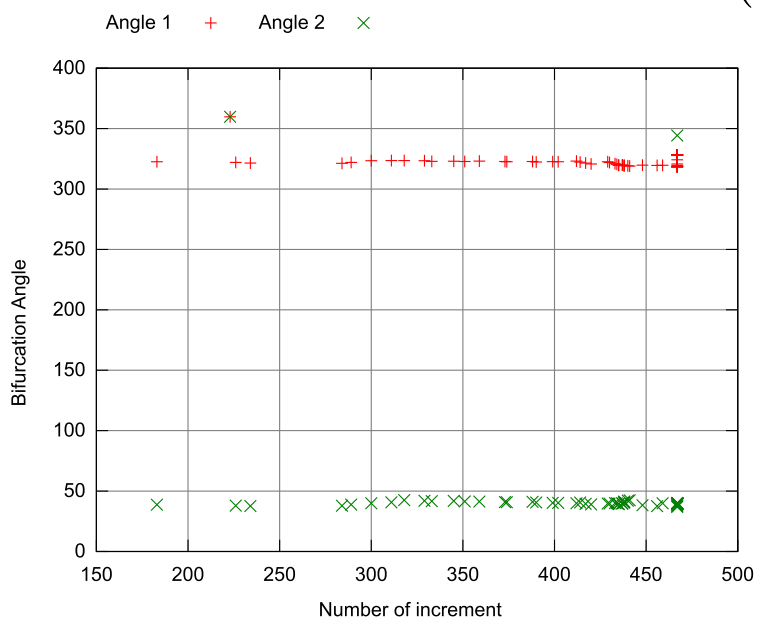

(c)

Fig. 25. (Application 2) Bifurcation angle using the proposed approach: (a) Mesh size 1 (coarse), (b) Mesh size 2 (medium), and (c) Mesh size 3 (fine).

In both applications, one can observe a strong dependence of the crack propagation path on the mesh size when using the element deletion based method, whereas the crack propagation direction is the same whatever is the mesh size when using the methodology proposed herein.

The reaction force history shows a dependence of the time at crack initiation, with a tendency to convergence for the methodology proposed. The drop in the reaction force during the crack propagation is relatively smooth for the element deletion based method and fast for the methodology proposed.

\subsection{Issues relative to the numerical algorithm}

There are two levels of numerical integration. At the initial-and boundary-value problem level, Abaqus implicit, integration solver was used. The algorithm is thus unconditionally stable. In parallel, for reason of simplicity, the elastic tangent operator was returned (from the user material subroutine) instead of the elastic-(visco) plastic-damage one. To favour convergence of the numerical results, time increments were chosen very small, leading to a slow convergence. Evolutions of time step and number of iterations before 


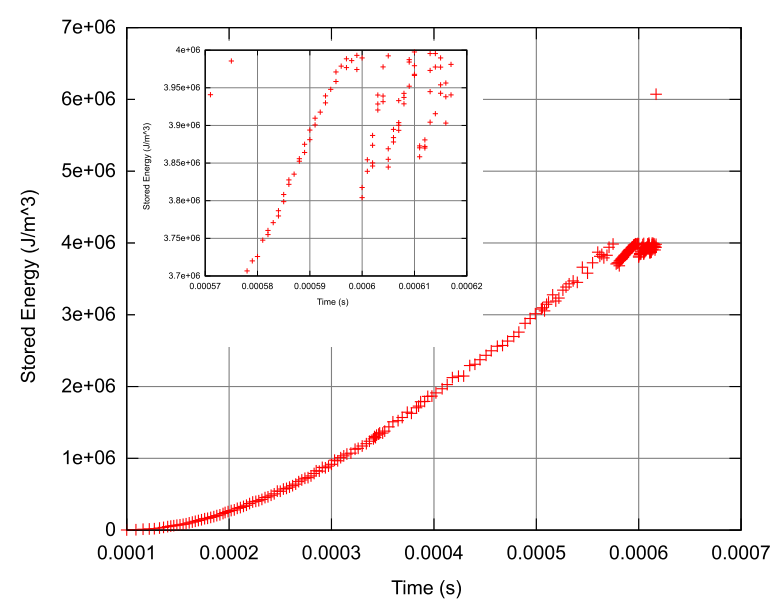

(a)

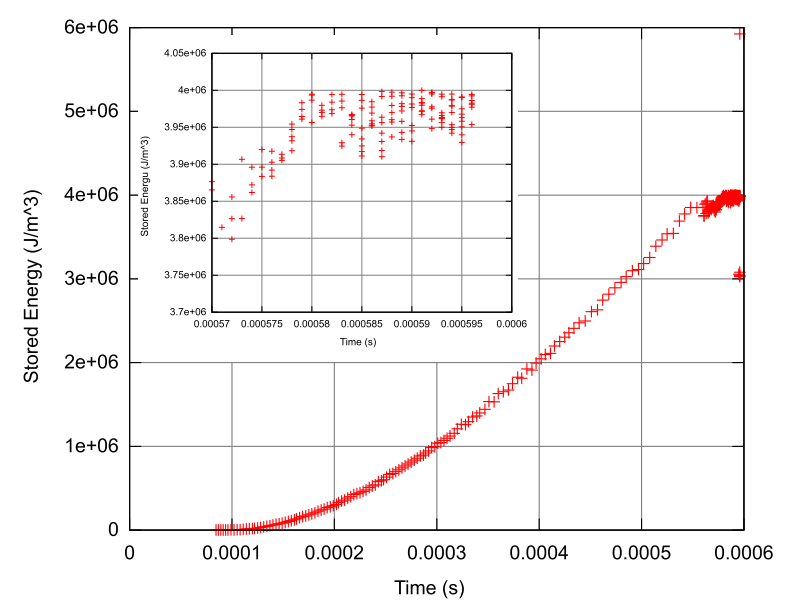

(b)

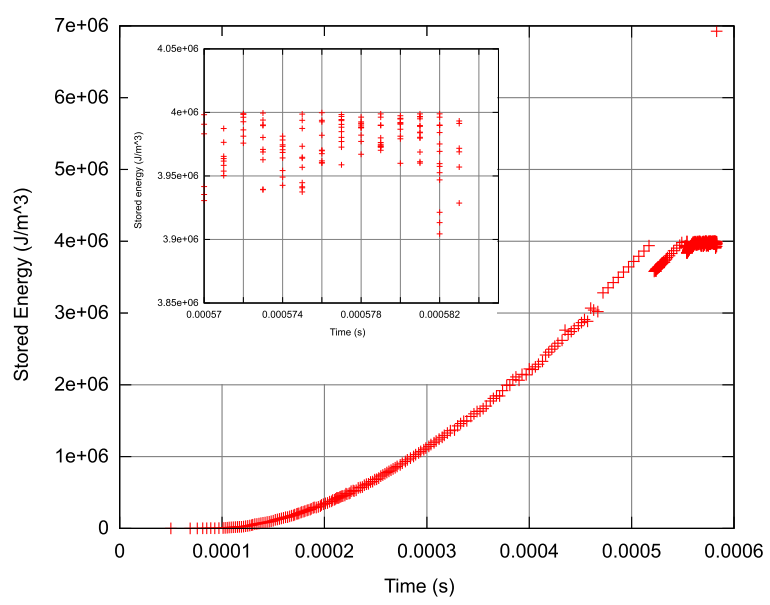

(c)

Fig. 26. (Application 2) Evolution of the stored energy using the proposed approach: (a) Mesh size 1 (coarse), (b) Mesh size 2 (medium), and (c) Mesh size 3 (fine).

convergence are drawn in Figs. 27 and 28, respectively, as a function of the increment number, during tension loaded asymmetric notched plate problem solving.

According to Fig. 27, one can distinguish three stages:

- stage Z1 (low increment number) during which the crack does not propagate and characterised by a time step close to $2.10^{-3} \mathrm{~ms}$

- stage Z2 (moderate increment number) during which the crack propagates and characterised by a time step close to $10^{-4} \mathrm{~ms}$,

- stage Z3 (large increment number) during which the crack crosses the structure and characterised by a time step close to $1 \mathrm{~ms}$.

Thus, solving the problem involves small time steps, see Fig. 27, and large numbers of iterations before convergence, see Fig. 28.

At the constitutive (Gurson) model level, as mentioned previously, numerical integration of the rate equations (in the user material subroutine) was achieved using the classical return mapping procedure combined with the Newton-Raphson solving algorithm, see $[28,29]$ for further details. 


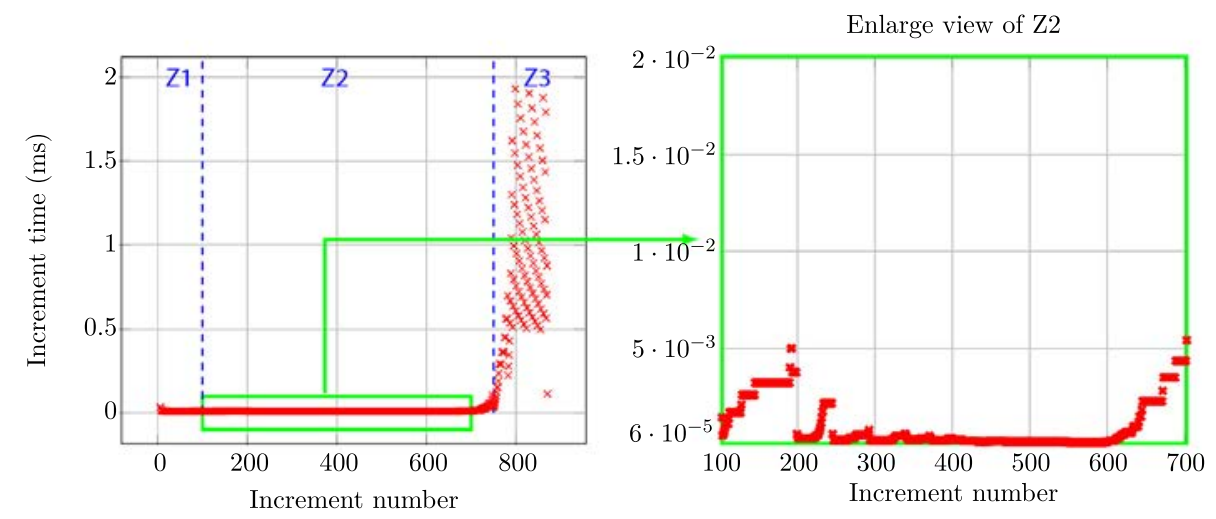

Fig. 27. Evolution of the time step during tension loaded asymmetric notched plate problem solving using mesh size 3 (fine): $\mathrm{Z} 1$ no crack propagation, $\mathrm{Z} 2$ crack propagation and $\mathrm{Z} 3$ failure of the structure.

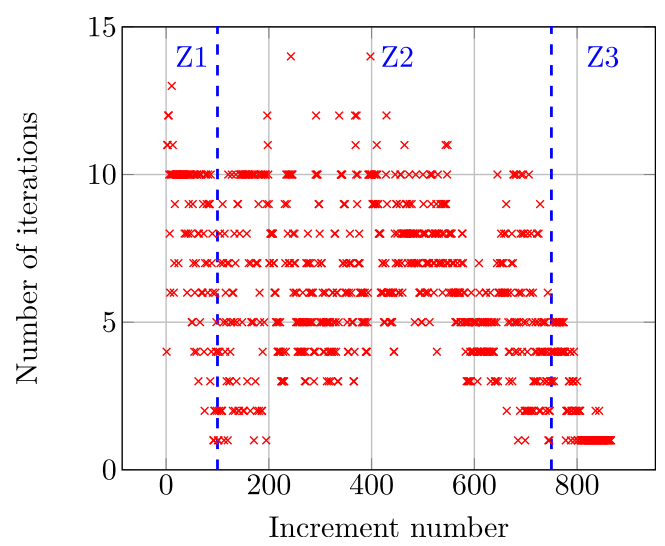

Fig. 28. Evolution of the number of iterations before convergence during tension loaded asymmetric notched plate problem solving using mesh size 3 (fine): Z1 no crack propagation, Z2 crack propagation and Z3 failure of the structure.

\section{Concluding remarks}

This work is devoted to the numerical simulation of crack propagation in engineering materials whose failure results from ductile damage.

The approach proposed in the present paper aims at coupling a GTN type strongly non linear constitutive model accounting for the combined effects of strain hardening, thermal softening, viscoplasticity and void growth induced damage, with the eXtended Finite Element Method within an engineering computation code, namely Abaqus, in view of describing the crack growth in structures.

In order to attenuate the mesh size dependence, all the quantities considered were averaged over a patch containing several elements and locating at the crack tip. Starting from the experimental evidence that the crack formation results from the deformation localisation during the phase of void coalescence, the bifurcation analysis was employed to identify the potential crack propagation orientations. The selection of the correct crack direction was achieved with respect to strain considerations. Considering that from a certain value of the stored energy cracking becomes the only mechanism able to accommodate extra deformation, we are assuming a crack propagation criterion involving the energy stored within the patch. The crack is consequently assumed to grow as long as both aforementioned conditions are satisfied: there exist potential crack propagation directions and the stored energy exceeds a critical value.

The methodology proposed has been applied to two configurations (crack containing plate and asymmetrically notched plate) and compared with the element deletion based method using the standard FEM 
formulation. In the case of the latter, the crack propagation is controlled by the maximum value of the porosity. The element deletion based method leads to a strong dependence of the crack propagation path on the mesh size, unlike the methodology proposed for which the crack propagation direction is the same whatever is the mesh size. The reaction force history shows a dependence of the time at crack initiation, with a tendency to convergence for the methodology proposed, and a drop during the crack propagation smoother for the element deletion based method.

A quantitative evaluation of the predictive ability of the methodology proposed, in terms of crack propagation direction, time at crack initiation and drop in the reaction force, has yet to be done regarding experimental results, the latter not being available at the moment.

\section{Acknowledgment}

The Authors would like to aknowledge the financial support of DGA (French Ministry of Defence) as well as the fruitful discussions with Prof. M. Salaun from Universite de Toulouse, ISAE/ICA, France, and Prof. A. Combescure from Université de Lyon, INSA-L/LaMCoS, France.

\section{Appendix A. Computation of the elastic-plastic tangent operator}

The following rates are expressed in the rotated frame. The stress rate second order tensor $\underset{\sim}{\dot{\sigma}}$ is defined by:

$$
\underset{\sim}{\dot{\sigma}}=\underset{\approx}{C}: \dot{\sim}_{\sim}^{e}
$$

The additive partition of the total strain rate $\underset{\sim}{\dot{\epsilon}}$ into elastic and plastic contributions, namely ${\underset{\sim}{e}}_{\sim}^{e}$ and $\dot{\epsilon}_{\sim}^{p}$, yields

$$
\dot{\epsilon}_{\sim}^{e}=\dot{\sim} \dot{\sim} \dot{\epsilon}^{p}
$$

Normality rule assumption leads to

$$
\dot{\epsilon}_{\sim}^{p}=\dot{\lambda} \frac{\partial \Phi_{*}}{\partial \underset{\sim}{\sigma}}
$$

where $\dot{\lambda}$ represents the plastic multiplier and $\Phi_{*}$ the modified GTN plastic potential, both in the frozen state.

$$
\Phi_{*}(\underset{\sim}{\sigma}, \kappa, T, f)=\left(\frac{\sigma_{e q}}{\bar{\sigma}_{y}}\right)^{2}+2 q_{1} f \cosh \left(-\frac{3}{2} q_{2} \frac{p_{m}+p_{r}}{{\overline{\sigma_{y}}}^{*}}\right)-\left(1+q_{3} f^{2}\right)
$$

with $\bar{\sigma}_{y} *$ the modified yield stress, with a rate independent yield stress part $\left(\sigma_{y}\right)$ similar to Eq. (21) and a rate dependent part $\left(\sigma_{y_{v p}}^{*}\right)$ which is viewed as a constant contribution.

$$
{\overline{\sigma_{y}}}^{*}(\kappa, T)=\sigma_{y}(\kappa, T)+\sigma_{y_{v p}}^{*}
$$

The consistency condition implies

$$
\dot{\Phi}_{*}\left(\underset{\sim}{\sigma}, \sigma_{y}, f\right)=\frac{\partial \Phi_{*}}{\partial \underset{\sim}{\sigma}}: \underset{\sim}{\dot{\sigma}}+\frac{\partial \Phi_{*}}{\partial \overline{\sigma_{y}}} \dot{\sigma_{y}} *+\frac{\partial \Phi_{*}}{\partial f} \dot{f}=0
$$

The yield stress rate ${\dot{\overline{\sigma_{y}}}}^{*}$, in the frozen state, is expressed by

$$
{\dot{\sigma_{y}}}^{*}=\dot{\sigma}_{y}=\frac{\partial \sigma_{y}}{\partial \kappa} \dot{\kappa}+\frac{\partial \sigma_{y}}{\partial T} \dot{T}
$$

We can reformulated $\dot{\kappa}$ and $\dot{T}$ (see Eq. (27) and (28)) as

$$
\dot{\kappa}=\frac{\underset{\sim}{\sigma}: \dot{\sim}_{\sim}^{p}}{(1-f) \overline{\sigma_{y}}}
$$




$$
\dot{T}=\frac{\underset{\sim}{\sigma}: \dot{\epsilon}_{\sim}^{p}-r \dot{\kappa}}{\rho C}
$$

Injecting equations [(A.1)-(A.3), (A.7)-(A.9)] into Eq. (A.6), we find

$$
\begin{aligned}
& \frac{\partial \Phi_{*}}{\partial \underset{\sim}{\sigma}}: \underset{\approx}{C}: \underset{\sim}{\dot{\epsilon}}=\frac{\partial \Phi_{*}}{\partial \underset{\sim}{\sigma}}: \underset{\approx}{C}: \dot{\lambda} \frac{\partial \Phi_{*}}{\partial \underset{\sim}{\sigma}}-\frac{\partial \Phi_{*}}{\partial f}(1-f) \operatorname{Tr}\left[\dot{\lambda} \frac{\partial \Phi_{*}}{\partial \underset{\sim}{\sigma}}\right]-\left(\frac{\partial \Phi_{*}}{\partial \overline{\sigma_{y}}}+B \frac{\partial \Phi_{*}}{\partial f}\right)\left(\frac{\partial \sigma_{y}}{\partial \kappa}\left(\frac{\underset{\sim}{\sigma}: \dot{\lambda} \frac{\partial \Phi_{*}}{\partial \sigma}}{(1-f) \dot{\sigma_{y}}}\right)\right. \\
& \left.+\frac{\partial \sigma_{y}}{\partial T}\left(\frac{\underset{\sim}{\sim}: \dot{\lambda} \frac{\partial \Phi_{*}}{\partial \sigma}}{\rho C}-\frac{r}{\rho C}\left(\frac{\underset{\sim}{\sigma}: \dot{\lambda} \frac{\partial \Phi_{*}}{\partial \underset{\sim}{\sigma}}}{(1-f) \dot{\sigma_{y}}}\right)\right)\right)
\end{aligned}
$$

allowing to express the multiplier $\dot{\lambda}$ as:

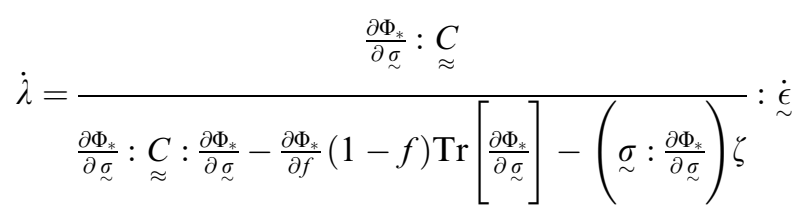

with $\zeta$ :

$$
\begin{aligned}
\zeta= & \frac{\partial \Phi_{*}}{\partial{\overline{\sigma_{y}}}^{*}}\left[\left(\frac{\partial \sigma_{y}}{\partial \kappa} \frac{1}{(1-f){\overline{\sigma_{y}}}^{*}}\right)+\frac{\partial \sigma_{y}}{\partial T}\left(\frac{1}{\rho C}-\frac{r}{\rho C} \frac{1}{(1-f){\overline{\sigma_{y}}}^{*}}\right)\right]+B \frac{\partial \Phi_{*}}{\partial f}\left[\left(\frac{\partial \sigma_{y}}{\partial \kappa} \frac{1}{(1-f){\overline{\sigma_{y}}}^{*}}\right)\right. \\
& \left.+\frac{\partial \sigma_{y}}{\partial T}\left(\frac{1}{\rho C}-\frac{r}{\rho C} \frac{1}{(1-f){\overline{\sigma_{y}}}^{*}}\right)\right]
\end{aligned}
$$

Starting from the following definition of the elastic-plastic tangent operator $\underset{\approx}{L}$

$$
\underset{\sim}{\dot{\sigma}}=\underset{\approx}{C}:\left(\underset{\sim}{\dot{\epsilon}}-\dot{\lambda} \frac{\partial \Phi_{*}}{\partial \underset{\sim}{\sigma}}\right)=\underset{\approx}{L}: \underset{\sim}{\dot{\epsilon}}
$$

one obtains

$$
\underset{\approx}{\sim} \underset{\approx}{C}-\frac{(\underset{\approx}{C}: \underset{\sim}{m}) \otimes(\underset{\sim}{m}: \underset{\approx}{C})}{\Omega}
$$

with $\underset{\sim}{m}$ and $\Omega$ expressed by

$$
\begin{aligned}
& \underset{\sim}{m}=\frac{\partial \Phi_{*}}{\partial \underset{\sim}{\sigma}} \\
& \Omega=\underset{\sim}{m}: \underset{\sim}{C}: \underset{\sim}{m}-\frac{\partial \Phi_{*}}{\partial f}(1-f) \operatorname{Tr}[\underset{\sim}{m}]-(\underset{\sim}{\sigma}: \underset{\sim}{m}) \zeta
\end{aligned}
$$

\section{Appendix B. Strain localisation plane identification}

According to Ortiz et al. [40] for two-dimensionnal cases, finding the strain localisation plane direction consists in solving

$$
f(x)=a_{4} x^{4}+a_{3} x^{3}+a_{2} x^{2}+a_{1} x+a_{0}=0
$$

where $x=\tan \theta$, with $\theta$ the angle of the localisation plane with respect to $x$-axis, and the coefficients $a_{i}$ being given by 


$$
\begin{aligned}
& a_{0}=L_{1111} L_{1212}-L_{1112} L_{1211} \\
& a_{1}=L_{1111} L_{1222}+L_{1111} L_{2212}-L_{1112} L_{2211}-L_{1122} L_{1211} \\
& a_{2}=L_{1111} L_{2222}+L_{1112} L_{1222}+L_{1211} L_{2212}-L_{1122} L_{1212}-L_{1122} L_{2211}-L_{1212} L_{2211} \\
& a_{3}=L_{1112} L_{2222}+L_{1211} L_{2222}-L_{1122} L_{2212}+L_{1222} L_{2211} \\
& a_{4}=L_{1212} L_{2222}-L_{2212} L_{1222}
\end{aligned}
$$

The normal $\underline{g}$ is accordingly

$$
\underline{g}=\left\{\begin{array}{c}
\cos \theta \\
\sin \theta
\end{array}\right\}
$$

As mentioned previously, the vector $g$, associated to the angle $\theta$, rending negative the value of the function $\mathrm{f}(\mathrm{x})$ is assumed to be the normal of the potential localisation plane and further crack growth direction.

\section{References}

[1] A.L. Gurson, Continuum theory of ductile rupture by void nucleation and growth: part I - Yield criteria and flow rules for porous ductile media, J. Eng. Mater. Technol. 99 (1977) 2-15.

[2] P. Perzyna, Stability of flow processes for dissipative solids with internal imperfections, J. Appl. Math. Phys. 35 (1984) $848-867$.

[3] J. Lemaitre, A continuous damage mechanics model for ductile fracture, J. Eng. Mater. Technol. 107 (1985) 83-89.

[4] G. Rousselier, Ductile fracture models and their potential in local approach of fracture, Nucl. Eng. Des. 105 (1987) $97-111$.

[5] M. Brünig, A framework for large strain elastic-palstic damage mechanics based on metric transformations, Int. J. Eng. Sci. 39 (2001) $1033-1056$.

[6] V. Tvergaard, A. Needleman, Analysis of the cup-cone fracture in a round tensile bar, Acta Metall. 32 (1984) $157-169$.

[7] R. Becker, A. Needleman, O. Richmon, V. Tvergaard, Void growth and failure in notched bars, J. Mech. Phys. Solids 36 (1988) $317-$ 351.

[8] J.B. Leblond, G. Perrin, J. Devaux, An improved Gurson-type model for hardenable ductile metals, Eur. J. Mech. Solids 14 (1995) 499-527.

[9] K. Nahshon, J.W. Hutchinson, Modification of the Gurson model for shear failure, Eur. J. Mech. Solids 27 (2008) 1-17.

[10] P. Longère, A.G. Geffroy, B. Leblé, A. Dragon, Modelling the transition between dense metal and damaged (micro-porous) metal viscoplasticity, Int. J. Damage Mech. 21 (2012) 1020-1063.

[11] M.E. Mear, J.W. Hutchinson, Influence of yield surface curvature on flow localization in dilatant plasticity, Mech. Mater. 4 (1985) 395-407.

[12] J. Besson, D. Steglich, W. Brocks, Modeling of crack growth in round bars and plane strain specimens, Int. J. Solids Struct. 38 (2001) 8259-8284.

[13] P.O. Bouchard, F. Bay, Y. Chastel, I. Tovena, Crack propagation modelling using an advanced remeshing technique, Comput. Methods Appl. Mech. Eng. 189 (2000) 723-742.

[14] N. Moes, J. Dolbow, T. Belytschko, A finite element method for crack growth without remeshing, Int. J. Numer. Methods Eng. 46 (1999) 131-150.

[15] J.M. Melenk, I. Babuska, The partition of unity finite element method: basic theory and applications, Comput. Methods Appl. Mech. Eng. 139 (1996) 289-314.

[16] D. Grégoire, H. Maigre, A. Combescure, New experimental and numerical techniques to study the arrest and the restart of a crack under impact in transparent materials, Int. J. Solids Struct. 46 (2009) 3480-3491.

[17] D. Haboussa, D. Grégoire, T. Elguedj, H. Maigre, A. Combescure, X-FEM analysis of the effects of holes or other cracks on dynamic crack propagations, Int. J. Numer. Methods Eng. 86 (2011) 618-636.

[18] D. Haboussa, T. Elguedj, B. Leblé, A. Combescure, Simulation of the shear-tensile mode transition on dynamic crack propagation, Int. J. Fract. 178 (2012) 195-213.

[19] P. Pourmodheji, M. Mashayekhi, Improvement of the extended finite element method for ductile crack growth, Mater. Sci. Eng. 551 (2012) 255-271.

[20] M.R.R. Seabra, P. Sustaric, J.M.A. Cesar de Sa, T. Rodic, Damage driven crack initiation and propagation in ductile metals using XFEM, Comput. Mech. 52 (2013) 161-179.

[21] P. Broumand, A.R. Khoei, The extended finite element method for large deformation ductile fracture problems with a non-local damage-plasticity model, Eng. Fract. Mech. 112-113 (2013) 97-125.

[22] E. Giner, N. Sukumar, J.E. Tarancón, F.J. Fuenmayor, An Abaqus implementation of the extended finite element method, Eng. Fract. Mech. 76 (2009) 347-368.

[23] P. Longère, A. Dragon, Description of shear failure in ductile metals via back stress concept linked to damage-microporosity softening, Eng. Fract. Mech. 98 (2013) 92-108.

[24] T. Elguedj, A. Gravouil, A. Combescure, Appropriate extended functions for X-FEM simulation of plastic fracture mechanics, Comput. Methods Appl. Mech. Eng. 195 (2006) 501-515. 
[25] A.E. Huespe, A. Needleman, J. Oliver, P.J. Sanchez, A finite thickness band method for ductile fracture analysis, Int. J. Plast. 25 (2009) 2349-2365.

[26] S. Graff, S. Forest, S. Strudel, J.L. Prioul, P. Pilvin, J.L. Béchade, Strain localization phenomena associated with static and dynamic strain ageing in notched specimens: experiments and finite element simulations, Mater. Sci. Eng. 387-389 (2004) 181-185.

[27] P. Longère, A. Dragon, Inelastic heat fraction evaluation for engineering problems involving dynamic plastic localization phenomena, J. Mech. Mater. Struct. 4 (2009) 319-349.

[28] N. Aravas, On the numerical integration of a class of pressure-dependent plasticity models, Int. J. Numer. Methods Eng. 24 (1987) $1395-1416$.

[29] G. Vadillo, R. Zaera, J. Fernandez-Saez, Consistent integration of the constitutive equations of Gurson materials under adiabatic conditions, Comput. Methods Appl. Mech. Eng. 197 (2008) 1280-1295.

[30] B. Prabel, A. Combescure, A. Gravouil, S. Marie, Level set X-FEM non-matching meshes: application to dynamic crack propagation in elastic-plastic media, Int. J. Numer. Methods Eng. 69 (2007) 1553-1569.

[31] G. Zi, T. Belytschko, New crack-tip elements for XFEM and applications to cohesive crack, Int. J. Numer. Methods Eng. 57 (2003) $2221-2240$.

[32] N. Moes, A. Gravouil, T. Belytschko, Non-planar 3D crack growth by the extended finite element and level sets - Part I: mechanical model, Int. J. Numer. Methods Eng. 53 (2002) 2549-2568.

[33] P.J. Sanchez, A.E. Huespe, J. Oliver, On some topics for the numerical simulation of ductile fracture, Int. J. Plast. 24 (2008) $1008-$ 1038.

[34] R. Hill, Acceleration waves in solids, J. Mech. Phys. Solids 10 (1962) 1-16.

[35] V. Kumar, R. Drathi, A meshless cracking particles approach for ductile fracture, J. Civ. Eng. 18 (2014) $238-248$.

[36] T. Rabczuk, T. Belytschko, A three dimensional large deformation meshfree method for arbitrary evolving cracks, Comput. Methods Appl. Mech. Eng. 196 (2007) 2777-2799.

[37] R. Hambli, Comparison between Lemaitre and Gurson damage models in crack growth simulation during blanking process, Int. J. Mech. Sci. 43 (2001) 2769-2790.

[38] W.B. Lievers, A.K. Pilkey, M.J. Worswick, The co-operative role of voids and shear bands in strain localization during bending, Mech. Mater. 35 (2003) 661-674.

[39] A.G. Geffroy, P. Longère, B. Leblé, Fracture analysis and constitutive modelling of ship structure steel behaviour regarding explosion, Eng. Fail. Anal. 18 (2011) 670-681.

[40] M. Ortiz, Y. Leroy, A. Needleman, A finite element method for localized failure analysis, Comput. Methods Appl. Mech. Eng. 61 (1987) 189-214. 